\title{
Genetic Predictors for Sinusoidal Obstruction Syndrome-A Systematic Review
}

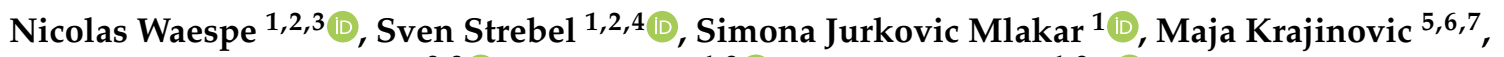 \\ Claudia Elisabeth Kuehni ${ }^{2,8}\left(\mathbb{D}\right.$, Tiago Nava 1,9 ${ }^{(D)}$ and Marc Ansari 1,9,*(D) \\ 1 CANSEARCH Research Platform in Pediatric Oncology and Hematology, University of Geneva, \\ 1205 Geneva, Switzerland; nicolas.waespe@ispm.unibe.ch (N.W.); sven.strebel@ispm.unibe.ch (S.S.); \\ simona.mlakar@unige.ch (S.J.M.); tiago.nava@unige.ch (T.N.) \\ 2 Institute of Social and Preventive Medicine, University of Bern, 3012 Bern, Switzerland; \\ claudia.kuehni@ispm.unibe.ch \\ 3 Graduate School for Cellular and Biomedical Sciences (GCB), University of Bern, 3012 Bern, Switzerland \\ 4 Graduate School for Health Sciences (GHS), University of Bern, 3012 Bern, Switzerland \\ 5 Charles-Bruneau Cancer Center, CHU Sainte-Justine Research Center, Department of Pediatrics, \\ Montreal, QC H3T 1C5, Canada; maja.krajinovic@umontreal.ca \\ 6 Clinical Pharmacology Unit, Department of Pediatrics, CHU Sainte-Justine, Montreal, QC H3T 1C5, Canada \\ 7 Department of Pharmacology, Faculty of Medicine, University of Montreal, Montreal, QC H3T 1J4, Canada \\ 8 Division of Pediatric Hematology/Oncology, Department of Pediatrics, Inselspital, Bern University Hospital, \\ University of Bern, 3012 Bern, Switzerland \\ 9 Department of Women, Children and Adolescents, Division of Pediatric Oncology and Hematology, \\ Geneva University Hospital, 1205 Geneva, Switzerland \\ * Correspondence: Marc.Ansari@hcuge.ch; Tel.: +41-79-553-6100
}

Citation: Waespe, N.; Strebel, S.; Jurkovic Mlakar, S.; Krajinovic, M.; Kuehni, C.E.; Nava, T.; Ansari, M. Genetic Predictors for Sinusoidal Obstruction Syndrome-A Systematic Review. J. Pers. Med. 2021, 11, 347. https://doi.org/10.3390/ jpm11050347

Academic Editor: Youssef Daali

Received: 12 March 2021

Accepted: 22 April 2021

Published: 26 April 2021

Publisher's Note: MDPI stays neutral with regard to jurisdictional claims in published maps and institutional affiliations.

Copyright: (c) 2021 by the authors. Licensee MDPI, Basel, Switzerland. This article is an open access article distributed under the terms and conditions of the Creative Commons Attribution (CC BY) license (https:/ / creativecommons.org/licenses/by/ $4.0 /)$.

\begin{abstract}
Sinusoidal obstruction syndrome (SOS) is a potentially life-threatening complication after hematopoietic stem cell transplantation (HSCT) or antineoplastic treatment without HSCT. Genetic variants were investigated for their association with SOS, but the evidence is inconclusive. We performed a systematic literature review to identify genes, gene variants, and methods of association analyses of genetic markers with SOS. We identified 23 studies after HSCT and 4 studies after antineoplastic treatment without HSCT. One study (4\%) performed whole-exome sequencing (WES) and replicated the analysis in an independent cohort, 26 used a candidate-gene approach. Three studies included $>200$ participants $(11 \%)$, and six were of high quality $(22 \%)$. Variants in 34 genes were tested in candidate gene studies after HSCT. Variants in GSTA1 were associated with SOS in three studies, MTHFR in two, and CPS1, CTH, CYP2B6, GSTM1, GSTP1, HFE, and HPSE in one study each. UGT2B10 and LNPK variants were identified in a WES analysis. After exposure to antineoplastic agents without HSCT, variants in six genes were tested and only GSTM1 was associated with SOS. There was a substantial heterogeneity of populations within and between studies. Future research should be based on sufficiently large homogenous samples, adjust for covariates, and replicate findings in independent cohorts.
\end{abstract}

Keywords: sinusoidal obstruction syndrome; genetic polymorphism; pharmacogenomic variants; genetic predisposition; genetic association studies; whole-exome sequencing; candidate gene analysis; hematopoietic stem cell transplantation; antineoplastic agents; systematic review

\section{Introduction}

Sinusoidal obstruction syndrome (SOS) of the liver is a serious, potentially lifethreatening complication occurring usually within the first 30 days after hematopoietic stem cell transplantation (HSCT) [1] or after treatment with some antineoplastic agents without HSCT [2]. Diagnostic criteria are based on clinical and laboratory findings including weight gain/ascites, hyperbilirubinemia, and hepatomegaly/right upper abdominal quadrant pain (Seattle criteria [3] and Baltimore criteria [4]). These were revised more recently to 
better reflect different forms of SOS presentation [5]. Depending on which criteria are used, the frequency of patients diagnosed with SOS might differ by two-fold [6]. Risk factors after HSCT include underlying disease (thalassemia major, leukemia, hemophagocytosis), pre-existing liver disease or injury, iron overload, laboratory markers (increased bilirubin and transaminases before HSCT), previous treatment with gemtuzumab ozogamicin, previous allogeneic $\mathrm{HSCT}$, high-intensity conditioning regimens, and total body irradiation $[5,7,8]$. Children are twice as likely to develop SOS after HSCT than adults [9] and even higher within the first two years of life [5]. Antineoplastic agents associated with SOS without HSCT are alkylating agents, platinum agents (particularly oxaliplatin [10,11]), and purine analogues [12-14]. SOS was also reported after acute lymphoblastic leukemia (ALL) induction treatment [13] and treatment with actinomycin D for nephroblastoma [15].

The pathogenesis of SOS is complex and includes damage to endothelial cells and hepatocytes. Cytotoxic agents and their metabolites lead to the activation of stress response mechanisms, and loss of integrity of the endothelial lining in the liver sinusoidal space [16]. Cytokines released by the damaged tissues further enhance the damaging process and activate the coagulation cascade leading to thrombi in the liver microvasculature [17]. All of these processes result in sinusoidal obstruction, liver cell injury, and hepato-renal syndrome associated with kidney failure, and death [18]. Obstruction of the sinusoidal spaces was found to be secondary to endothelial damage with inflammation and locally activated coagulation with an increase in procoagulant factors and a decrease in antithrombotic proteins. Subsequently, venous outflow obstruction of the liver causes damage to the liver cells $[19,20]$. The molecular mechanisms thought to affect SOS include the cytochrome P-450 enzymatic system, which plays an important role in the clearance of toxic metabolites of chemotherapeutics (e.g., cyclophosphamide) and the glutathione pathway, which is involved in metabolizing busulfan. Inflammatory response and activation of coagulation with release of von Willebrand factor, plasminogen activator inhibitor-1 (PAI1), and thrombomodulin were reported to contribute to disease progression [21].

Defibrotide is the only approved treatment for severe SOS. Defibrotide stabilizes endothelial cell homeostasis by reducing endothelial-cell activation and damage. It also reduces the plasma levels of plasminogen activator inhibitor-1 (PAI-1), and results in the restoration of the thrombo-fibrinolytic balance [20]. Defibrotide has been successfully used as prophylaxis in patients deemed to be at an increased risk for SOS due to pre-existing liver disease, an underlying condition, or treatment factors [9]. Prophylactic ursodeoxycholic acid has shown efficacy in the reduction in SOS and mortality [1].

While the underlying molecular mechanisms are still incompletely understood, genetic variants have been postulated to influence the incidence of SOS for the last two decades [22]. Various pathways have been assessed for their association with SOS. A recent systematic review looked at the influence of glutathione S-transferase genes on pharmacokinetic parameters of busulfan and SOS incidence [23]. To our knowledge, there is no systematic review that summarizes the evidence for all postulated germline genetic predictors for sinusoidal obstruction syndrome. This systematic review describes all identified publications that investigated gene variants associated with SOS in patients of any age who underwent HSCT or were exposed to antineoplastic agents without HSCT. We describe genes and gene variants that were identified and the respective association analyses that were used.

\section{Materials and Methods}

\subsection{Study Design}

We performed a systematic literature review following the Preferred Reporting Items for Systematic Review and Meta-Analysis (PRISMA 2009) statement [24] (Supplementary Table S1). We pre-registered the research protocol on PROSPERO (CRD42020215568). 


\subsection{Study Selection: Eligibility Criteria}

We included studies reporting on humans of any age undergoing (allogeneic and autologous) HSCT or treatment with antineoplastic agents without HSCT. We defined antineoplastic agents as all treatments targeting malignant neoplasms including steroids, antihormones, and monoclonal antibodies. We did not use language restrictions. We included studies published from 1 January 1980 to 24 September 2020.

We selected observational studies and longitudinal interventional trials. We initially retained reviews to screen references and then excluded them from the final analysis if no original data were reported. We also excluded opinions, commentaries, conference abstracts, case reports or case series reporting on less than 20 participants, and all reports without original data. We further excluded studies reporting on animal and cell models, and in silico (computer-model) analyses only.

\subsection{Outcome Definition: Sinusoidal Obstruction Syndrome}

We searched for studies with SOS as either the main outcome or outcome with a dedicated association analysis. For patients undergoing HSCT, we included studies with the outcome "sinusoidal obstruction syndrome" as defined by the authors and identified those using established criteria (using the Seattle, ref. [3,8] Baltimore, ref. [4] or new EBMT guidelines [5]). We included patients exposed to antineoplastic agents with the outcome "sinusoidal obstruction syndrome" as defined by the authors. We evaluated the criteria used to diagnose SOS and attributed quality scores (see below: Quality assessment and risk of bias).

\subsection{Exposures: Genetic Variants}

We searched for studies that reported germline genetic variants and their effect on SOS occurrence (i.e., that compared patients with a specific genetic variant to those without).

\subsection{Identification of Studies}

A systematic literature search was performed using (a) PubMed, (b) EMBASE, (c) Web of Science (Core Collection), (d) Cochrane, (e) CINAHL (EBSCO), and (f) Google Scholar. We searched (g) clinicaltrials.gov for registered studies and searched for published results. We performed a search of the references of identified manuscripts to retrieve further literature. We removed duplicates in the process using the citation manager EndNote (version X8) and Rayyan (https:/ / rayyan.qcri.org; accessed on 1 October 2020) [25].

The search strategy was built for all databases using Medical Subject Headings (MeSH) and Title/Abstract (TiAb) terms. We restricted the population of interest to "humans". For the outcome, we searched for "sinusoidal obstruction syndrome", and related terms. For the exposure, we used "hematopoietic stem cell transplantation" or "antineoplastic agents" and related terms. For the prognostic factor, we used "genetic variation", "pharmacogenomic variants", "pharmacogenetics", and related terms (see Supplementary Table S2 for the detailed search strategy). We performed the last search update on 24 September 2020. We did not search for unpublished data.

\subsection{Study Selection}

Two authors independently evaluated the eligibility for the inclusion of the identified manuscripts by (i) screening all titles and abstracts, excluding obviously not fitting manuscripts, and then (ii) performing a full-text review of the remaining manuscripts to check for eligibility. For all manuscripts with a discordant assessment of eligibility, the two authors sought agreement through discussion, and where no agreement was reached, a third author judged on eligibility. We used Rayyan for the screening of titles and abstracts.

\subsection{Data Extraction}

One author extracted data from texts, tables, and graphs. A second author checked the accuracy and completeness of data. Any disagreements were resolved by discussion, 
and where no agreement was reached, by arbitration of a third author. Unclear or missing data were requested from the corresponding author of the respective manuscripts. We designed a data collection form that included information on the authors; the manuscript; the methodology of germline DNA sequencing and associated quality measures; the study population; the statistical analysis; and results including the strength of association such as relative risks, odds ratios, and hazard ratios. The form was developed and discussed within the review group before full data extraction.

\subsection{Quality Assessment and Risk of Bias}

The quality assessment was performed by two authors independently using an adapted scoring table based on a previously published scoring system [26] that used the STrengthening the REporting of Genetic Association Studies (STREGA) guidelines [27], an extension of the STROBE Statement [28]. We based the assessment on the reporting, external and internal validity, confounding bias, selection bias, and study power. A study that scored 6 or more out of the 12 points was regarded as high-quality (Supplementary Table S3).

\section{Results}

\subsection{Study Identification and Selection}

We identified 708 unique citations after the removal of duplicates and excluded 677 manuscripts after the title and abstract screening. After assessment of the full text, two reports on subpopulations $[29,30]$ of larger studies were excluded as well as one that assessed HSCT donor genotypes [31] and one review without original data [23]. Finally, we retained 27 manuscripts (Figure 1).

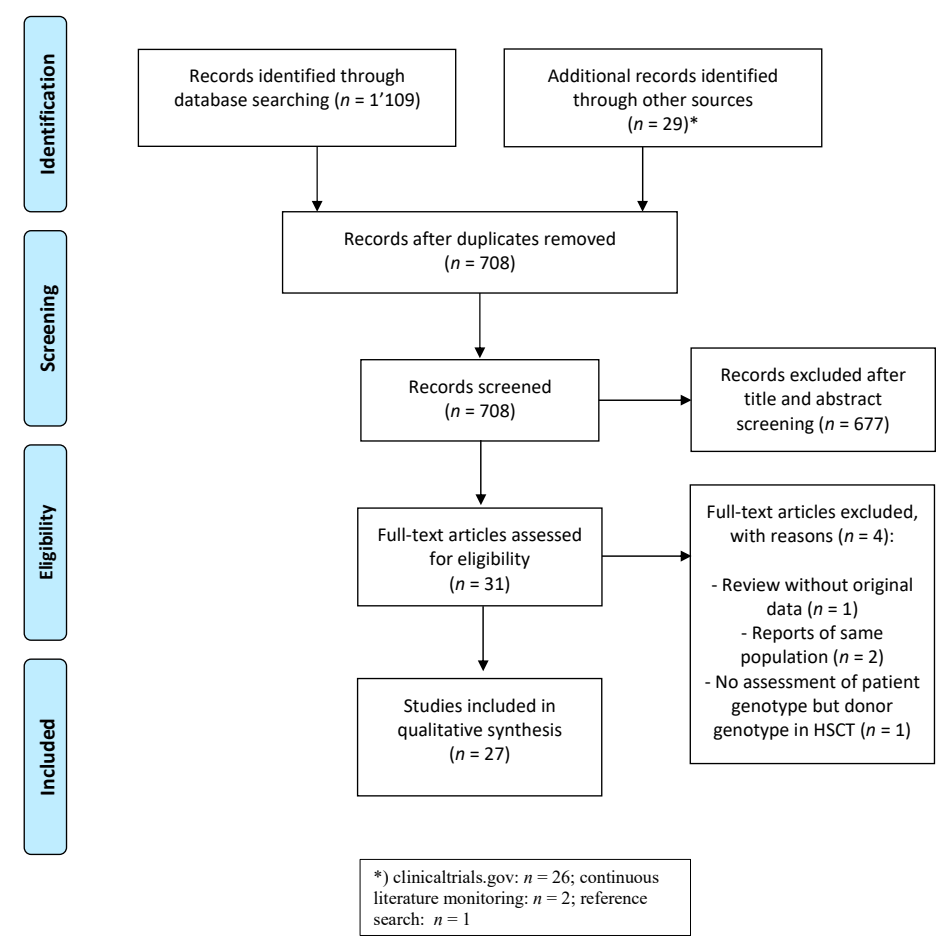

Figure 1. PRISMA Flowchart of the literature review and selection process for genetic predictors for sinusoidal obstruction syndrome after hematopoietic stem cell transplantation and antineoplastic treatment exposure.

\subsection{Characteristics of Included Studies}

\subsubsection{Study Characteristics}

Of the 27 retained manuscripts, 23 were original articles (85\%), 2 were short reports $(7 \%)[13,32]$, and 2 were letters $(7 \%)$ [22,33]. The study populations (for summary statistics, 
see Table 1$)$ were collected from retrospective cohort studies $(n=23,85 \%)$, two prospective trials $(7 \%)[12,13]$ and two case-control studies (7\%) [22,32]. Eleven manuscripts included patients from Europe (41\%), nine from the US or Canada (33\%), four from Asia (15\%), two from Israel $(7 \%)$, and one from Turkey (4\%). Twenty studies reported data from a single institution (74\%), six from multiple sites $(22 \%)$ and one did not specify clearly $(4 \%)$.

Table 1. Summary characteristics of studies reporting on genetic predictors of SOS $(n=27)$.

\begin{tabular}{|c|c|c|}
\hline Characteristics & $n$ & Proportion (\%) \\
\hline \multicolumn{3}{|l|}{ Centers included } \\
\hline monocentric & 20 & 74.1 \\
\hline multicentric & 6 & 22.2 \\
\hline unclear & 1 & 3.7 \\
\hline \multicolumn{3}{|l|}{ Location } \\
\hline Europe & 11 & 40.7 \\
\hline North America & 9 & 33.3 \\
\hline Asia & 4 & 14.8 \\
\hline Others & 3 & 11.1 \\
\hline \multicolumn{3}{|l|}{ Study design } \\
\hline cohort & 23 & 85.2 \\
\hline prospective trial & 2 & 7.4 \\
\hline case-control & 2 & 7.4 \\
\hline \multicolumn{3}{|l|}{ Sample size } \\
\hline median, IQR (n) & 84 & $65-142$ \\
\hline $0-50$ & 3 & 11.1 \\
\hline $51-100$ & 13 & 48.1 \\
\hline $101-150$ & 4 & 14.8 \\
\hline $151-200$ & 4 & 14.8 \\
\hline 201 and more & 3 & 11.1 \\
\hline \multicolumn{3}{|l|}{ Age group at treatment } \\
\hline children and adolescents only & 10 & 37 \\
\hline children, adolescents, and adults & 11 & 40.7 \\
\hline adults only & 6 & 22.2 \\
\hline \multicolumn{3}{|l|}{ Treatment exposure } \\
\hline allogeneic HSCT, busulfan-based & 10 & 37 \\
\hline allogeneic HSCT, various regimens & 9 & 33.3 \\
\hline autologous and allogeneic HSCT & 4 & 14.8 \\
\hline non-HSCT & 4 & 14.8 \\
\hline \multicolumn{3}{|l|}{ Outcome } \\
\hline incidence cohort-based samples (mean $\%$, range $\%$ ) & 16.5 & $2.3-42.9$ \\
\hline (modified) Seattle criteria & 15 & 55.6 \\
\hline Baltimore criteria & 5 & 18.5 \\
\hline other criteria/unspecified & 7 & 25.9 \\
\hline \multicolumn{3}{|l|}{ Association analysis } \\
\hline candidate gene analysis & 26 & 96.3 \\
\hline genome/exome wide analysis & 1 & 3.7 \\
\hline
\end{tabular}

Legend: HSCT, hematopoietic stem cell transplantation; IQR, interquartile range; $n$, number.

\subsubsection{Population}

Overall, 3150 genotyped patients were included among all the studies. The study population size varied from 18 to 351 with a median of 84 . Three studies (11\%) included more than 200 genotyped patients $[12,13,34]$. Eleven studies reported on a mixed population of children, adolescents, and adults (41\%); 10 studies reported on children/adolescents $(37 \%)$; and 6 reported on adult patients $(22 \%)$. The median age of participants ranged 
from 4 to 62 years. The proportion of females ranged from $28 \%$ to $61 \%$ (average $52 \%$ ). The ethnicity of the study populations was only described in 14 manuscripts (52\%), rendering a classification into ethnic groups of the whole population impossible.

\subsubsection{Treatment Exposure}

Twenty-three studies reported on patients who underwent HSCT (85\%). Of those, 19 restricted the exposure to allogeneic HSCT (83\%), while four also included autologous HSCT (17\%) [22,35-37]. Four studies included only HSCT from a sibling donor $(17 \%)$ [38-41], the others included various donor types. A wide array of underlying diagnoses were included in most studies on HSCT $(n=18,78 \%)$, whereas five studies included only selected diagnoses (two included only acute myeloid leukemia [33,37], two thalassemia [41,42], and one different types of leukemia [40]). Standardized prophylactic treatment was used in four studies $(17 \%)[33,37,43,44]$, while seven $(30 \%)$ studies used prophylactic treatment for SOS only in a subgroup: one mentioned ursodeoxycholic acid only (4\%) [43]; one heparin only [44]; one defibrotide only [45]; two studies mentioned ursodeoxycholic acid and heparin (9\%) [33,37]; one ursodeoxycholic acid and defibrotide [46]; and one study all three prophylactic treatments [47]. It is unclear whether studies not mentioning SOS prophylaxis did not administer prophylaxis or did not describe its use.

Four studies included participants exposed to antineoplastic agents without HSCT $(15 \%)$. All studies focused on a single underlying disease: two studied acute lymphoblastic leukemia (50\%) [12,13], one acute myeloid leukemia (25\%) [32], and one colorectal cancer (25\%) [48]. No prophylactic treatment was described.

\subsubsection{Genotyping}

Most studies used a candidate gene approach genotyping based on pre-specified genes $(n=26,96 \%)$, only one recent study employed exome-wide sequencing. This is also the only study that attempted to replicate findings in an independent cohort [47]. Six (22\%) studies reported on the quality of genotyping by mentioning the number of successful genotyping attempts or cross-validation with a different technique and $11(41 \%)$ took into account Hardy-Weinberg equilibrium when reporting the results.

\subsubsection{Outcome}

SOS incidence across all cohort-based studies was $16.5 \%$ (range $2.3 \%$ to $42.9 \%$ ). The definition of SOS was based on the Seattle or modified Seattle criteria in half of the studies $(n=15,56 \%)$, and the Baltimore criteria in five (19\%). Various other criteria were used in six studies (22\%): two used either clinical criteria or histopathological criteria [22,32], one only histopathological criteria [48], one the National Cancer Institute Common Toxicity Criteria (CTC) versions 2 and 3 [49], and two other clinical criteria based on previously published data $[13,44]$. One study did not clearly specify the criteria used $(4 \%)$ [50].

\subsection{Quality of Studies and Publication Bias}

Using an adapted scoring based on the STREGA guidelines, we identified six (22\%) studies that we ranked of high quality. The median score of all studies was 5 (range: 2-9 points, Supplementary Table S4). Most studies $(n=25,93 \%)$ described clearly their population with exposure and outcome definitions. While all studies reported on the origin of the study population, only two $(7 \%)$ studies stratified results by study population origin/ethnicity [13,35]. Two studies (7\%) performed a power analysis [46] (one of them performed this post hoc [13]). One study replicated the findings in an independent cohort [47]. Clinical characteristics potentially associated with the outcome were described in 15 studies (56\%) and 11 (41\%) adjusted the genotype-phenotype analysis for clinical variables. 


\subsection{Investigated Genes for Association with SOS after HSCT}

\subsubsection{Glutathione S-Transferase}

In the 23 studies that included patients with SOS after HSCT (Table 2), variants in 34 different genes were tested, including eight genes identified in the discovery dataset for the WES analysis and tested in the independent replication cohort [47]. The most frequent genes were from the glutathione S-transferase family: GSTA1 and GSTM1 (9 studies each), GSTP1 (7 studies), and GSTT1 (6 studies). Other members of the glutathione S-transferase family were only investigated in one study each (GSTO1, GSTO2, and GSTZ1). GSTA1 and GSTM1 variants were inconsistently associated with SOS: two pediatric studies by Ansari et al. from 2017 and 2020 (replication cohort) reported associations with a slow metabolizer haplotype group of GSTA1 diplotypes (as defined by the presence of any combination with the *B1b haplotype, ${ }^{*} \mathrm{~B} 1 \mathrm{a} * \mathrm{~B} 1 \mathrm{a}$, or $\left.{ }^{*} \mathrm{~B} 2{ }^{*} \mathrm{~B} 1 \mathrm{a}\right)[47,51]$ with odds ratios (OR) of 9.0 (95\%-confidence interval [CI] 2.6-31) and 3.1 (CI 1.2-8.0), respectively. The study by Curtis et al. from 2016 performed a gene-gene interaction study with $C T H$ but also compared the GSTA1* $\mathrm{B}^{*} \mathrm{~B}$ diplotypes to ${ }^{*} \mathrm{~A}{ }^{*} \mathrm{~A} /{ }^{*} \mathrm{~A}{ }^{*} \mathrm{~B}$ diplotypes and reported an OR of 10.9 (CI 2.3-51.3) [46]. The *B haplotype, corresponding to the rs3957357 (C > T) or -69 variant, was not found to be associated with SOS in two studies with 84 and 55 adult patients [36,52], and three pediatric and one mainly adult study with 29 to 77 participants $[37,41,53,54]$.

The homozygous deletion of GSTM1 often referred to as "null genotype" was associated with SOS after allogeneic HSCT with a busulfan-based conditioning regimen in pediatric beta-thalassemia patients (OR 4.3, CI 1.5-12.5, $p=0.008$ ) published by Srivastava et al. [42]. This association was not replicated in subsequent studies in predominantly adult $[36,37,40,52]$ and pediatric cohorts $[41,51,54]$, with one pediatric study by Zwaveling et al. showing possible evidence of association (no OR reported, $p=0.07$ ) [53]. The GSTP1 rs1695 (A > G) variant was associated with SOS in a study by Krivoy et al. [37] of 63 adult patients undergoing HSCT for acute myeloid leukemia (no OR reported, $p=0.05$ ) but not in other predominantly adult $[36,40,52]$ or pediatric studies $[51,53,54]$. The GSTP1 rs1138272(C > T) variant was tested in two studies and the rs614080 $(A>G)$ variant in one study without showing evidence for an association. The GSTT1 "null genotype" was not associated with SOS in two pediatric [42,53] and four mainly adult [36,37,40,52] studies. GSTO1, GSTO2, and GSTZ1 variants were not found to be associated with SOS [36].

\subsubsection{Cytochrome $\mathrm{P} 450$}

Cytochrome P450 family genes were the second group of genes frequently assessed in included studies. CYP2B6 is an important enzyme in the bioactivation of cyclophosphamide and the ${ }^{*} 6$ haplotype corresponding to $\operatorname{rs} 3745274(\mathrm{G}>\mathrm{T})$ and $\operatorname{rs} 2279343(\mathrm{~A}>\mathrm{G})$, was associated with SOS in a study by Rocha et al. (OR 3.5, CI 1.1-10.9) [40]. This study included predominantly adult leukemia patients undergoing HSCT with different regimens, which included cyclophosphamide in $82 \%$. Two other studies including mostly patients treated with cyclophosphamide-containing regimens [36,43] did not find the same association in the CYP2B $6 * 6$ haplotype or other assessed variants ( $5 \mathrm{~A}$ haplotype $=\operatorname{rs} 3211371(C>T), \operatorname{rs2279344}(A>G), \operatorname{rs} 2099361(A>C), r s 8100458(C>T)$, rs2014141(A > G)). Further variants assessed by Rocha et al. [40] were not associated with SOS $\left({ }^{*} 2 \mathrm{~A}\right.$ haplotype $=\operatorname{rs} 8192709(\mathrm{C}>\mathrm{T}),{ }^{*} 4$ haplotype $=\mathrm{rs} 2279343(\mathrm{~A}>\mathrm{G}), * 5$ haplotype $=$ rs3211371 $(C>T))$. Variants in CYP2C19 [34], which is an important enzyme in cyclophosphamide metabolism, and CYP2C9 [34,36,43], which has a possible role in busulfan metabolite metabolism, were investigated in a number of studies but no associations were identified. Variants in the ATP-binding cassette subfamily B, member 1 ( $A B C B 1)$, also called multidrug resistance-1 (MDR1) gene, were included in two predominantly adult studies without association $[37,40]$. 


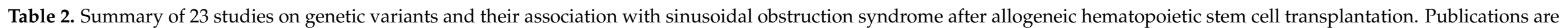
listed in chronological order of publication.

\begin{tabular}{|c|c|c|c|c|c|c|c|c|c|}
\hline $\begin{array}{l}\text { Lead Author, } \\
\text { Journal Year }\end{array}$ & Study Design & Location & $\begin{array}{c}\text { Population } \\
\text { (Diagnoses, Age) }\end{array}$ & Exposure, Location & $n$ (SOS/Total) & $\begin{array}{l}\text { Genes/ } \\
\text { Region }\end{array}$ & $\begin{array}{c}\text { Variants } \\
\text { Investigated }\end{array}$ & OR/ RR (CI) & $p$-Value \\
\hline \multirow{2}{*}{$\begin{array}{l}\text { Duggan C, et al. } \\
\text { Bone Marrow } \\
\text { Transplant. } \\
\text { 1999. [22] }\end{array}$} & \multirow{2}{*}{$\begin{array}{l}\text { Candidate-gene; } \\
\text { case-control }\end{array}$} & \multirow{2}{*}{$\begin{array}{c}\text { St James's } \\
\text { Hospital and } \\
\text { Trinity College } \\
\text { Dublin, Ireland }\end{array}$} & \multirow{2}{*}{$\begin{array}{l}\text { Unclear diagnoses, } \\
\text { median age } 29 \text { years } \\
\quad \text { (range } 4-55)\end{array}$} & \multirow{2}{*}{$\begin{array}{c}\text { AlloHSCT and } \\
\text { autoHSCT with } \\
\text { various regimens }(\mathrm{Bu} \text {, } \\
\mathrm{Cy}, \mathrm{Mel}, \mathrm{TBI} \text {, others) }\end{array}$} & \multirow{2}{*}{$\begin{array}{l}\text { 22/287 }(7.7 \%) \\
\text { genotyped: } \\
15 / 51(29.4 \%)\end{array}$} & $F 2$ & $\begin{array}{l}\text { rs1799963(GA } \\
\text { vs. GG) }\end{array}$ & - & $p=0.05$ \\
\hline & & & & & & F5 & $\begin{array}{l}\text { rs6025(GG vs. } \\
\text { AG/AA) }\end{array}$ & - & $p=0.05$ \\
\hline \multirow{7}{*}{$\begin{array}{l}\text { Pihusch M, et al. } \\
\text { Transplantation. } \\
\text { 2004 [44] }\end{array}$} & \multirow{7}{*}{$\begin{array}{c}\text { Candidate-gene; } \\
\text { cohort }\end{array}$} & \multirow{7}{*}{$\begin{array}{l}\text { José-Carreras } \\
\text { transplantation } \\
\text { unit Munich, } \\
\text { Germany }\end{array}$} & \multirow{7}{*}{$\begin{array}{l}\text { Various malignant } \\
\text { and non-malignant } \\
\text { diagnoses; median } \\
\text { age } 43 \text { years } \\
\text { (range 14-62) }\end{array}$} & \multirow{7}{*}{$\begin{array}{c}\text { AlloHSCT with } \\
\text { various regimens }(\mathrm{Bu}, \\
\text { Cy, Mel, TBI, others) }\end{array}$} & \multirow{7}{*}{$3 / 89(3.4 \%)$} & $F 2$ & rs1799963(G > A) & "no effect" & - \\
\hline & & & & & & F5 & $\operatorname{rs} 6025(G>A)$ & "no effect" & - \\
\hline & & & & & & MTHFR & rs1801133(C > T) & "no effect" & - \\
\hline & & & & & & ITGB3 & $\operatorname{rs} 591(C>T)$ & "no effect" & - \\
\hline & & & & & & $F G B$ & rs $1800790(\mathrm{G}>\mathrm{A})$ & "no effect" & - \\
\hline & & & & & & SERPINE1 & $\begin{array}{l}\text { rs1799889 } \\
\text { (4G allele) }\end{array}$ & $\begin{array}{c}(83.3 \% \text { vs. } \\
55.1 \%)\end{array}$ & NS \\
\hline & & & & & & $A C E$ & $\begin{array}{l}\text { rs1799752 } \\
\text { (D allele) }\end{array}$ & "no effect" & - \\
\hline $\begin{array}{l}\text { Srivastava } \\
\text { A, et al. Blood. } \\
2004 \text { [42] }\end{array}$ & $\begin{array}{c}\text { Candidate-gene; } \\
\text { cohort }\end{array}$ & $\begin{array}{l}\text { Hôpital Robert } \\
\text { Debré, Paris, } \\
\text { France }\end{array}$ & $\begin{array}{c}\text { Beta-thalassemia } \\
\text { major; median age } 6 \\
\text { years (range 2-16) }\end{array}$ & $\begin{array}{c}\text { Busulfan- } \\
\text { cyclophosphamide- } \\
\text { based } \\
\text { alloHSCT }\end{array}$ & $33 / 114(28.9 \%)$ & GSTT1 & $\begin{array}{c}\text { "null } \\
\text { genotype" } \ddagger\end{array}$ & $\begin{array}{c}\text { OR 0.6 } \\
(0.2-1.9) \dagger\end{array}$ & $\mathrm{p}=0.4 \dagger$ \\
\hline \multirow[t]{2}{*}{$\begin{array}{l}\text { Kallianpur } \\
\text { AR et al. Bone } \\
\text { Marrow } \\
\text { Transplant. } \\
2005 \text { [35] }\end{array}$} & \multirow[t]{2}{*}{$\begin{array}{c}\text { Candidate-gene; } \\
\text { cohort }\end{array}$} & \multirow[t]{2}{*}{$\begin{array}{c}\text { Multicentric, } \\
\text { two centers in } \\
\text { Nashville, } \\
\text { Tennessee, USA }\end{array}$} & \multirow[t]{2}{*}{$\begin{array}{l}\text { Various } \\
\text { hematological and } \\
\text { solid neoplasms; } \\
\text { mean age } 44 \text { years } \\
\text { (range 19-64) }\end{array}$} & \multirow[t]{2}{*}{$\begin{array}{c}\text { AlloHSCT and } \\
\text { autoHSCT with } \\
\text { various regimens (Bu, } \\
\text { Cy, TBI, others) }\end{array}$} & \multirow[t]{2}{*}{$30 / 166(18.1 \%)$} & HFE & $\operatorname{rs} 1800562(A>G)$ & $\begin{array}{c}\text { RR 3.7 } \\
(1.2-12.1) ; \text { RR } \\
\text { 1.7 (0.4-6.8) for } \\
\text { heterozygotes; } \\
\text { RR 8.6 (1.5-48.5) } \\
\text { for } \\
\text { homozygotes }+\end{array}$ & $p=0.01 t$ \\
\hline & & & & & & CPS1 & $\begin{array}{l}\text { rs7422339(CC } \\
\text { vs. AC/AA) }\end{array}$ & - & $p=0.038$ \\
\hline
\end{tabular}


Table 2. Cont.

\begin{tabular}{|c|c|c|c|c|c|c|c|c|c|}
\hline $\begin{array}{l}\text { Lead Author, } \\
\text { Journal Year }\end{array}$ & Study Design & Location & $\begin{array}{c}\text { Population } \\
\text { (Diagnoses, Age) }\end{array}$ & Exposure, Location & $n$ (SOS/Total) & $\begin{array}{l}\text { Genes/ } \\
\text { Region }\end{array}$ & $\begin{array}{c}\text { Variants } \\
\text { Investigated }\end{array}$ & OR/ RR (CI) & $p$-Value \\
\hline $\begin{array}{l}\text { Elmaagacli } \\
\text { AH, et al. Bone } \\
\text { Marrow } \\
\text { Transplant. } \\
2007 \text { [34] }\end{array}$ & $\begin{array}{l}\text { Candidate-gene; } \\
\text { cohort }\end{array}$ & $\begin{array}{c}\text { University } \\
\text { Hospital of } \\
\text { Essen, Germany }\end{array}$ & $\begin{array}{c}\text { Various } \\
\text { hematological } \\
\text { neoplasms incl. } \\
\text { lymphomas; median } \\
\text { age } 41 \text { years } \\
\text { (range 17-67) }\end{array}$ & $\begin{array}{l}\text { AlloHSCT with } \\
\text { various regimens }(\mathrm{Bu}, \\
\mathrm{Cy}, \mathrm{TBI} \text {, others) }\end{array}$ & $20 / 286(7 \%)$ & CYP2C19 & $\begin{array}{c}\text { Poor vs. } \\
\text { intermedi- } \\
\text { ate/extensive } \\
\text { metabolizers } \\
\text { (rs4244285(AA } \\
\text { vs. AG/GG) } \\
\text { rs4986893(AA } \\
\text { vs. AG/GG)) }\end{array}$ & - & NS \\
\hline \multirow{5}{*}{$\begin{array}{c}\text { Goekkurt } \\
\text { E, et al. } \\
\text { Anticancer Res. } \\
2007 \text { [52] }\end{array}$} & \multirow{5}{*}{$\begin{array}{l}\text { Candidate-gene; } \\
\text { cohort }\end{array}$} & \multirow{5}{*}{$\begin{array}{l}\text { University } \\
\text { Hospital } \\
\text { Hamburg, } \\
\text { Germany }\end{array}$} & \multirow{5}{*}{$\begin{array}{c}\text { Various } \\
\text { hematological } \\
\text { malignancies and } \\
\text { non-malignant } \\
\text { diagnoses; median } \\
\text { age 39.5 years } \\
\text { (range 16-59) }\end{array}$} & \multirow{5}{*}{$\begin{array}{l}\text { Busulfan- } \\
\text { cyclophosphamide- } \\
\text { based } \\
\text { alloHSCT }\end{array}$} & \multirow{5}{*}{$36 / 84(42.9 \%)$} & GSTA1 & $\begin{array}{l}{ }^{*} \text { B vs. }{ }^{*} \mathrm{~A} \\
\text { haplotypes }\end{array}$ & - & NS \\
\hline & & & & & & GSTM1 & $\begin{array}{c}\text { "null } \\
\text { genotype" } \ddagger\end{array}$ & - & NS \\
\hline & & & & & & GSTP1 & $\operatorname{rs} 1695(A>G)$ & - & NS \\
\hline & & & & & & GSTT1 & $\begin{array}{c}\text { "null } \\
\text { genotype" } \ddagger\end{array}$ & - & NS \\
\hline & & & & & & MTHFR & $\operatorname{rs} 1801131(A>C)$ & $\begin{array}{c}\text { OR 9.4 } \\
(1.1-81.9)+\end{array}$ & $p=0.048+$ \\
\hline \multirow{2}{*}{$\begin{array}{l}\text { Kim I, et al. } \\
\text { Annals of } \\
\text { Hematol. } \\
2007 \text { [38] }\end{array}$} & \multirow[t]{2}{*}{$\begin{array}{c}\text { Candidate-gene; } \\
\text { cohort }\end{array}$} & \multirow{2}{*}{$\begin{array}{l}\text { Seoul National } \\
\text { University } \\
\text { College of } \\
\text { Medicine, South } \\
\text { Korea }\end{array}$} & \multirow{2}{*}{$\begin{array}{l}\text { Hematological } \\
\text { malignancies and } \\
\text { aplastic anemia; } \\
\text { median age } 36 \text { year } \\
\text { (range 16-52) }\end{array}$} & \multirow{2}{*}{$\begin{array}{c}\text { AlloHSCT with } \\
\text { various regimens }(\mathrm{Bu} \text {, } \\
\text { Cy, TBI) }\end{array}$} & \multirow[t]{2}{*}{$11 / 72(15.3 \%)$} & \multirow[t]{2}{*}{ MTHFR } & rs1801133(C > T) & - & $p=0.4$ \\
\hline & & & & & & & rs1801131 $(\mathrm{A}>\mathrm{C})$ & - & $p=0.48$ \\
\hline $\begin{array}{l}\text { Lee KH, et al. } \\
\text { Haematologica. } \\
2007 \text { [39] }\end{array}$ & $\begin{array}{c}\text { Candidate-gene; } \\
\text { cohort }\end{array}$ & $\begin{array}{l}\text { Seoul National } \\
\text { University } \\
\text { Hospital, South } \\
\text { Korea }\end{array}$ & $\begin{array}{l}\text { Hematological } \\
\text { malignancies incl. } \\
\text { lymphomas and } \\
\text { aplastic anemia; } \\
\text { median age } 40 \text { years } \\
\text { (range 16-70) }\end{array}$ & $\begin{array}{l}\text { AlloHSCT with } \\
\text { various regimens }(\mathrm{Bu}, \\
\text { Cy, Mel, TBI, others) } \\
\text { from HLA-matched } \\
\text { sibling donors }\end{array}$ & $19 / 152(12.5 \%)$ & P2RX7 & rs3751143(A > C) & - & $p=0.78$ \\
\hline
\end{tabular}


Table 2. Cont.

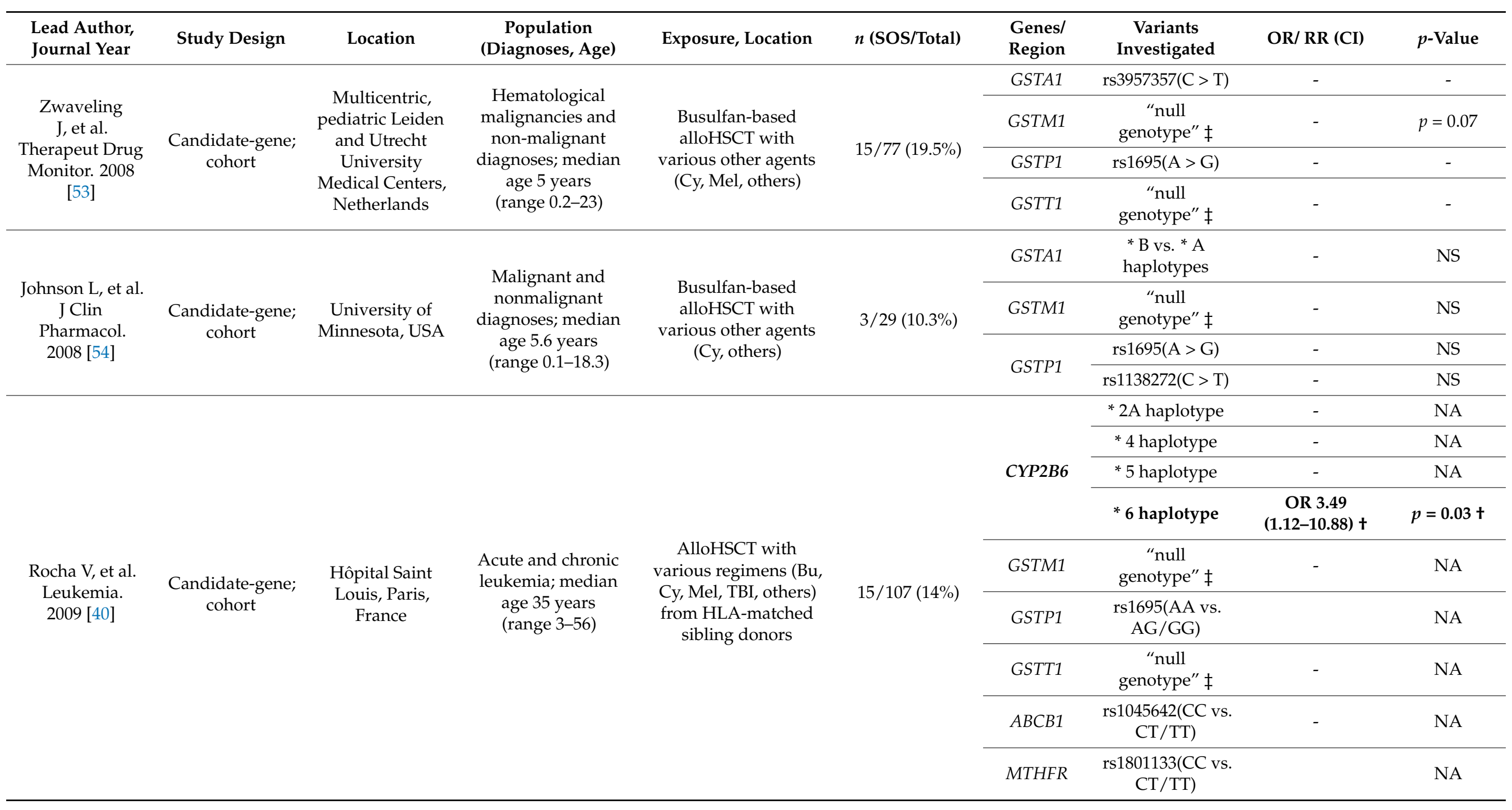


Table 2. Cont.

\begin{tabular}{|c|c|c|c|c|c|c|c|c|c|}
\hline $\begin{array}{l}\text { Lead Author, } \\
\text { Journal Year }\end{array}$ & Study Design & Location & $\begin{array}{c}\text { Population } \\
\text { (Diagnoses, Age) }\end{array}$ & Exposure, Location & $n$ (SOS/Total) & $\begin{array}{l}\text { Genes/ } \\
\text { Region }\end{array}$ & $\begin{array}{c}\text { Variants } \\
\text { Investigated }\end{array}$ & OR/ RR (CI) & $p$-Value \\
\hline & & & & & & & Apal (rs7975232) & - & NA \\
\hline & & & & & & $V D R$ & $\begin{array}{c}\text { BsmI } \\
\text { (rs1544410) }\end{array}$ & & NA \\
\hline & & & & & & & TaqI (rs731236) & - & NA \\
\hline $\begin{array}{l}\text { Elbahlawan } \\
\text { L, et al. J Ped } \\
\text { Hem Oncol. } \\
2012 \text { [49] }\end{array}$ & $\begin{array}{l}\text { Candidate-gene; } \\
\text { cohort }\end{array}$ & $\begin{array}{c}\text { St Jude } \\
\text { Children's } \\
\text { Research } \\
\text { Hospital, USA }\end{array}$ & $\begin{array}{c}\text { Malignant and } \\
\text { non-malignant } \\
\text { diagnoses; median } \\
\text { age 10.1 years } \\
\text { (range 1-19.6) }\end{array}$ & $\begin{array}{l}\text { AlloHSCT with } \\
\text { various regimens (Bu, } \\
\text { Cy, TBI, others) from } \\
\text { HLA-matched donors }\end{array}$ & $5 / 76(6.6 \%)$ & IL1B & $\operatorname{rs} 16944(A>G)$ & - & $p=0.18$ \\
\hline $\begin{array}{l}\text { Sucak GT, et al. } \\
\text { Ann } \\
\text { Hematology. } \\
2012 \text { [50] }\end{array}$ & $\begin{array}{l}\text { Candidate-gene; } \\
\text { cohort }\end{array}$ & $\begin{array}{l}\text { Gazi University, } \\
\text { Ankara, Turkey }\end{array}$ & $\begin{array}{l}\text { Malignant and } \\
\text { non-malignant } \\
\text { diagnoses; median } \\
\text { age } 27.5 \text { years } \\
\text { (range 16-64) }\end{array}$ & $\begin{array}{l}\text { AlloHSCT with } \\
\text { various regimens (Bu, } \\
\text { Mel, TBI, others) }\end{array}$ & $22 / 102(21.6 \%)$ & HFE & rs1799945(C > G) & - & $p>0.05$ \\
\hline \multirow{5}{*}{$\begin{array}{l}\text { Krivoy N, et al. } \\
\text { Curr Drug } \\
\text { Safety. } 2012 \text { [37] }\end{array}$} & \multirow{5}{*}{$\begin{array}{c}\text { Candidate-gene; } \\
\text { cohort }\end{array}$} & \multirow{5}{*}{$\begin{array}{l}\text { Technion-Israel } \\
\text { Institute of } \\
\text { Technology; } \\
\text { Haifa, Israel }\end{array}$} & \multirow{5}{*}{$\begin{array}{l}\text { Acute myeloid } \\
\text { leukemia; median } \\
\text { age } 39.2 \text { years } \\
\text { (SD 12.3) }\end{array}$} & \multirow{5}{*}{$\begin{array}{c}\text { Busulfan- } \\
\text { cyclophosphamide- } \\
\text { based autoHSCT and } \\
\text { alloHSCT }\end{array}$} & \multirow{5}{*}{$8 / 63(12.7 \%)$} & & rs1045642(C > T) & - & NS \\
\hline & & & & & & GSTA1 & rs3957357(C > T) & - & NS \\
\hline & & & & & & GSTM1 & $\begin{array}{c}\text { "null } \\
\text { genotype" } \ddagger\end{array}$ & - & NS \\
\hline & & & & & & GSTP1 & $\operatorname{rs1695}(A>G)$ & - & $p=0.05$ \\
\hline & & & & & & GSTT1 & $\begin{array}{c}\text { "null } \\
\text { genotype" } \ddagger\end{array}$ & - & NS \\
\hline \multirow{5}{*}{$\begin{array}{l}\text { Uppugunduri } \\
\text { CRS, et al. Phar- } \\
\text { macogenom J. } \\
2014[43]\end{array}$} & \multirow{5}{*}{$\begin{array}{c}\text { Candidate-gene; } \\
\text { cohort }\end{array}$} & \multirow{5}{*}{$\begin{array}{l}\text { CHU } \\
\text { Sainte-Justine, } \\
\text { Montreal, } \\
\text { Canada }\end{array}$} & \multirow{5}{*}{$\begin{array}{c}\text { Malignant and } \\
\text { non-malignant } \\
\text { diagnoses; median } \\
\text { age } 6.9 \text { years } \\
\text { (range } 0.1-19.9 \text { ) }\end{array}$} & \multirow{5}{*}{$\begin{array}{c}\text { Busulfan-based } \\
\text { alloHSCT with } \\
\text { various other agents } \\
\text { (Cy, Mel, TBI, others) }\end{array}$} & \multirow{5}{*}{$8 / 66(12.1)$} & \multirow{2}{*}{ CYP2B6 } & rs3211371(C > T) & - & NS \\
\hline & & & & & & & rs3745274(G > T) & - & NS \\
\hline & & & & & & \multirow{2}{*}{ CYP2C19 } & rs4244285(G > A) & - & NS \\
\hline & & & & & & & rs12248560 $(\mathrm{C}>\mathrm{T})$ & - & NS \\
\hline & & & & & & CYP2C9 & rs1799853(C > T) & - & NS \\
\hline
\end{tabular}


Table 2. Cont.

\begin{tabular}{|c|c|c|c|c|c|c|c|c|c|}
\hline $\begin{array}{l}\text { Lead Author, } \\
\text { Journal Year }\end{array}$ & Study Design & Location & $\begin{array}{c}\text { Population } \\
\text { (Diagnoses, Age) }\end{array}$ & Exposure, Location & $n$ (SOS/Total) & $\begin{array}{l}\text { Genes/ } \\
\text { Region }\end{array}$ & $\begin{array}{c}\text { Variants } \\
\text { Investigated }\end{array}$ & OR/ RR (CI) & $p$-Value \\
\hline & & & & & & CYP2C9 & rs1057910(G > A) & - & NS \\
\hline & & & & & & & rs2266780(A > G) & - & NS \\
\hline & & & & & & FMO3 & rs2266782(G > A) & - & NS \\
\hline & & & & & & & rs1736557(A > G) & - & NS \\
\hline \multirow{2}{*}{$\begin{array}{c}\text { Efrati E, et al. } \\
\text { Bone Marrow } \\
\text { Transplant. } \\
2014 \text { [33] }\end{array}$} & \multirow{2}{*}{$\begin{array}{c}\text { Candidate-gene; } \\
\text { cohort }\end{array}$} & \multirow{2}{*}{$\begin{array}{l}\text { Technion-Israel } \\
\text { Institute of } \\
\text { Technology; } \\
\text { Haifa, Israel }\end{array}$} & \multirow{2}{*}{$\begin{array}{c}\text { Acute myeloid } \\
\text { leukemia; adult } \\
\text { cohort }\end{array}$} & \multirow{2}{*}{$\begin{array}{c}\text { Busulfan- } \\
\text { cyclophosphamide- } \\
\text { based alloHSCT (with } \\
\text { TBI in one) }\end{array}$} & \multirow{2}{*}{$9 / 62(15 \%)$} & \multirow{2}{*}{ MTHFR } & $\begin{array}{l}\text { rs1801133(CC } \\
\text { vs. CT/TT) }\end{array}$ & - & $p=0.0096$ \\
\hline & & & & & & & $\begin{array}{c}\text { rs1801131(CC } \\
\text { vs. AC/AA) }\end{array}$ & - & $p=0.0002$ \\
\hline \multirow{3}{*}{$\begin{array}{l}\text { Seifert C, et al. J. } \\
\text { Cancer Res. } \\
\text { Clin. Oncol. } \\
2015 \text { [45] }\end{array}$} & \multirow{3}{*}{$\begin{array}{l}\text { Candidate-gene; } \\
\text { cohort }\end{array}$} & \multirow{3}{*}{$\begin{array}{c}\text { Jena University } \\
\text { Hospital, } \\
\text { Germany }\end{array}$} & \multirow{3}{*}{$\begin{array}{c}\text { Malignant and } \\
\text { non-malignant } \\
\text { diagnoses; median } \\
\text { age } 14 \text { years, } \\
\text { (range } 0-29 \text { ) }\end{array}$} & \multirow{3}{*}{$\begin{array}{c}\text { AlloHSCT with } \\
\text { various regimens }(\mathrm{Bu}, \\
\mathrm{Cy}, \mathrm{Mel}, \mathrm{TBI})\end{array}$} & \multirow{3}{*}{$12 / 160(7.5 \%)$} & \multirow{3}{*}{ HPSE } & $\begin{array}{c}\text { rs4693608(AA } \\
\text { vs. AG/GG) }\end{array}$ & - & $p=0.038$ \\
\hline & & & & & & & $\begin{array}{c}\text { rs4364254(TT } \\
\text { vs. TC/CC) }\end{array}$ & - & $p=0.004$ \\
\hline & & & & & & & $\begin{array}{c}\text { rs4693608(AA) } \\
\text { and } \\
\text { rs4364254(TT) † }\end{array}$ & $\begin{array}{c}4.06 \\
(1.14-14.4)+\end{array}$ & $p=0.03+$ \\
\hline \multirow{2}{*}{$\begin{array}{l}\text { Ansari M, et al. } \\
\text { Bone Marrow } \\
\text { Transplant. } \\
\text { 2016 [41] }\end{array}$} & \multirow[t]{2}{*}{$\begin{array}{c}\text { Candidate-gene; } \\
\text { cohort }\end{array}$} & \multirow[t]{2}{*}{$\begin{array}{c}\text { San Raffaele } \\
\text { Institute, Milan, } \\
\text { Italy }\end{array}$} & \multirow{2}{*}{$\begin{array}{c}\text { Thalassemia } \\
\text { intermedia }(20.5 \%) \\
\text { and thalassemia } \\
\text { major }(79.5 \%) ; \\
\text { median age } 8 \text { years } \\
\text { (range } 1.5-17)\end{array}$} & \multirow{2}{*}{$\begin{array}{c}\text { Busulfan- } \\
\text { cyclophosphamide- } \\
\text { based alloHSCT from } \\
\text { HLA-matched } \\
\text { sibling donors }\end{array}$} & \multirow[t]{2}{*}{$1 / 44(2.3 \%)$} & GSTA1 & $\begin{array}{c}{ }^{*} \mathrm{~B} \text { vs. }{ }^{*} \mathrm{~A} \\
\text { haplotypes } \\
\text { using } \\
\text { rs3957357(C > T) }\end{array}$ & - & NS \\
\hline & & & & & & GSTM 1 & $\begin{array}{c}\text { "null } \\
\text { genotype" } \ddagger\end{array}$ & - & NS \\
\hline $\begin{array}{l}\text { Byun JM, et al. } \\
\text { PloS One. } \\
2016 \text { [55] }\end{array}$ & $\begin{array}{l}\text { Candidate-gene; } \\
\text { cohort }\end{array}$ & $\begin{array}{l}\text { Seoul National } \\
\text { University } \\
\text { Hospital, South } \\
\text { Korea }\end{array}$ & $\begin{array}{l}\text { Hematological } \\
\text { malignancies incl. } \\
\text { lymphomas and } \\
\text { aplastic anemia; } \\
\text { median age 37.8 } \\
\text { years (SD 12.5) }\end{array}$ & $\begin{array}{l}\text { AlloHSCT with } \\
\text { various regimens (not } \\
\text { further specified) }\end{array}$ & $10 / 177(5.6 \%)$ & MTHFR & $\begin{array}{l}\text { rs1801133(TT vs. } \\
\text { CT/CC) }\end{array}$ & - & $p=0.234$ \\
\hline
\end{tabular}


Table 2. Cont.

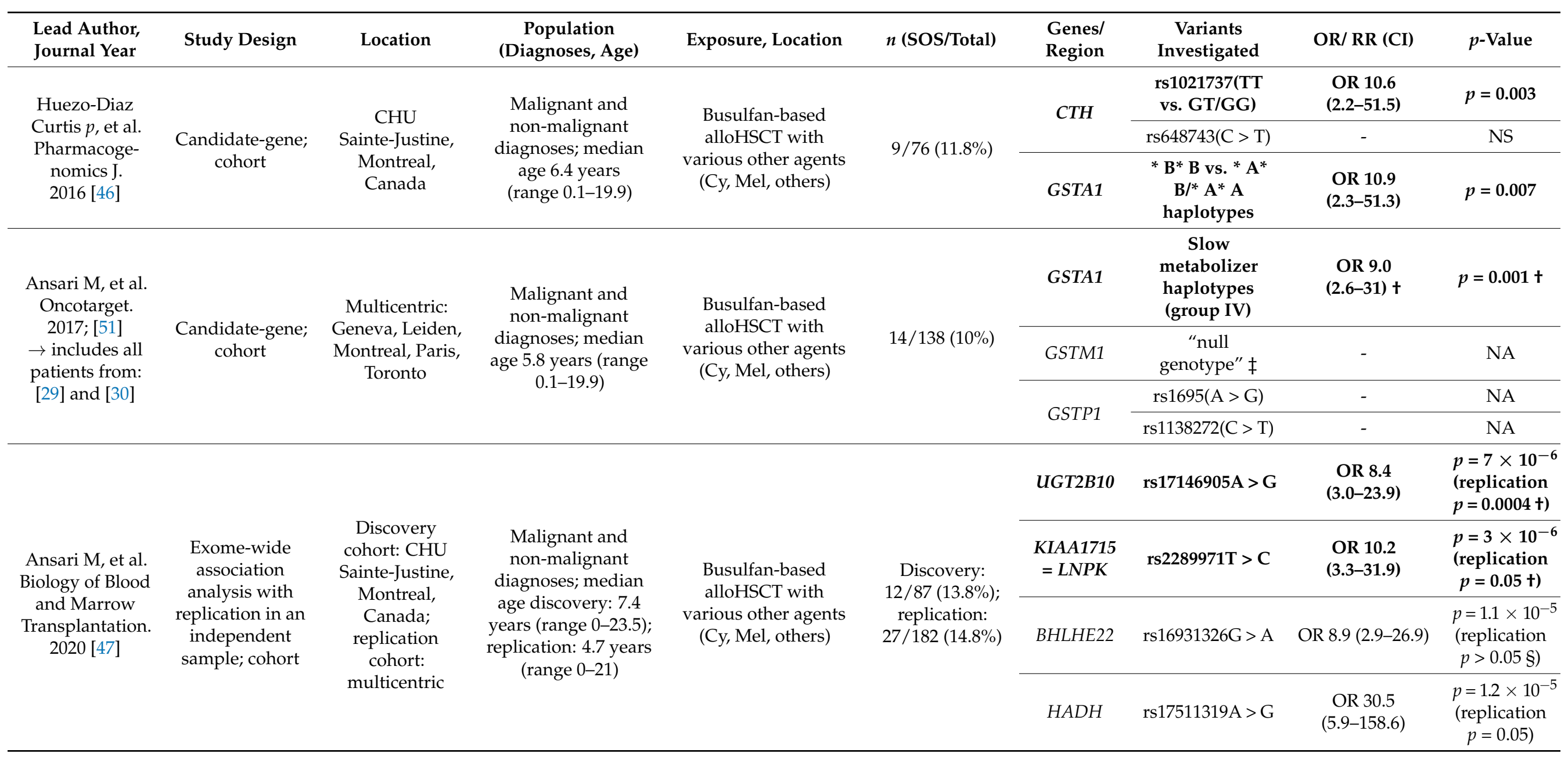


Table 2. Cont.

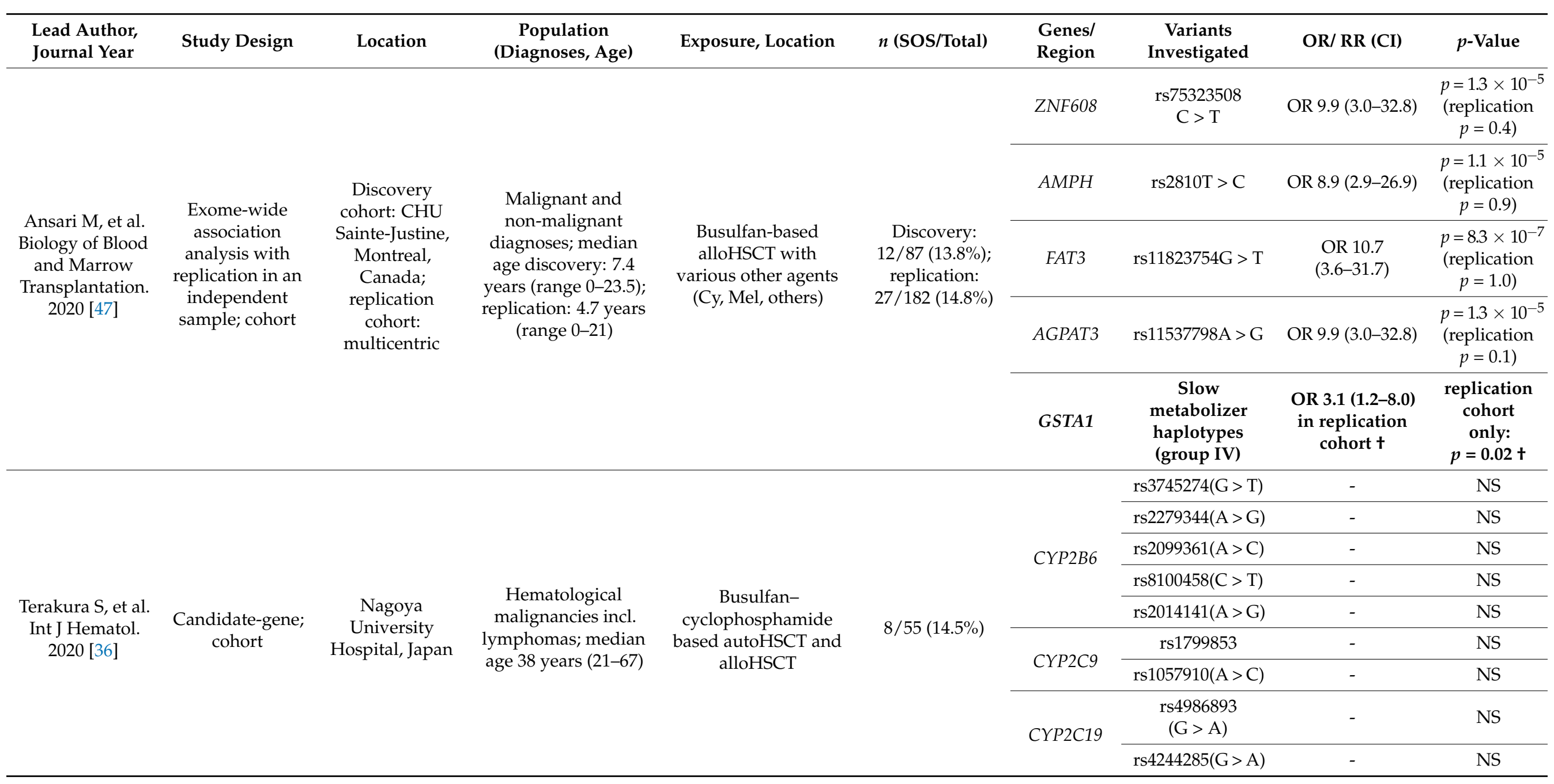


Table 2. Cont.

\begin{tabular}{|c|c|c|c|c|c|c|c|c|c|}
\hline $\begin{array}{l}\text { Lead Author, } \\
\text { Journal Year }\end{array}$ & Study Design & Location & $\begin{array}{c}\text { Population } \\
\text { (Diagnoses, Age) }\end{array}$ & Exposure, Location & $n$ (SOS/Total) & $\begin{array}{l}\text { Genes/ } \\
\text { Region }\end{array}$ & $\begin{array}{c}\text { Variants } \\
\text { Investigated }\end{array}$ & OR/ RR (CI) & $p$-Value \\
\hline \multirow{8}{*}{$\begin{array}{l}\text { Terakura S, et al. } \\
\text { Int J Hematol. } \\
2020[36]\end{array}$} & \multirow{8}{*}{$\begin{array}{c}\text { Candidate-gene; } \\
\text { cohort }\end{array}$} & \multirow{8}{*}{$\begin{array}{c}\text { Nagoya } \\
\text { University } \\
\text { Hospital, Japan }\end{array}$} & \multirow{8}{*}{$\begin{array}{c}\text { Hematological } \\
\text { malignancies incl. } \\
\text { lymphomas; median } \\
\text { age } 38 \text { years (21-67) }\end{array}$} & \multirow{8}{*}{$\begin{array}{c}\text { Busulfan- } \\
\text { cyclophosphamide } \\
\text { based autoHSCT and } \\
\text { alloHSCT }\end{array}$} & \multirow{8}{*}{$8 / 55(14.5 \%)$} & GSTA1 & $\begin{array}{l}{ }^{*} \text { B vs. }{ }^{*} \mathrm{~A} \\
\text { haplotype } \\
\text { (rs4715326) }\end{array}$ & - & NS \\
\hline & & & & & & GSTM1 & $\begin{array}{c}\text { "null } \\
\text { genotype" } \ddagger\end{array}$ & - & NS \\
\hline & & & & & & GSTO1 & rs4925(A > C) & - & NS \\
\hline & & & & & & \multirow{2}{*}{ GSTO2 } & rs156697(A > G) & - & NS \\
\hline & & & & & & & rs2297235(A > G) & - & NS \\
\hline & & & & & & \multirow{2}{*}{ GSTP1 } & $\operatorname{rs} 1695(\mathrm{~A}>\mathrm{G})$ & - & NS \\
\hline & & & & & & & rs614080((A > G) & - & NS \\
\hline & & & & & & GSTT1 & $\begin{array}{c}\text { "null } \\
\text { genotype" } \ddagger\end{array}$ & - & NS \\
\hline
\end{tabular}

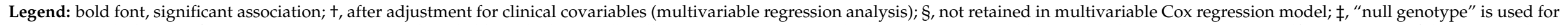

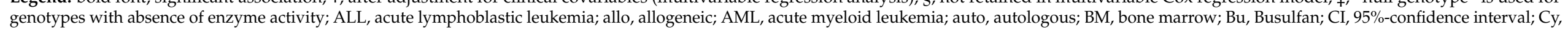

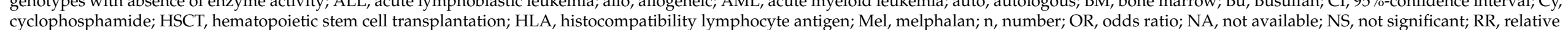

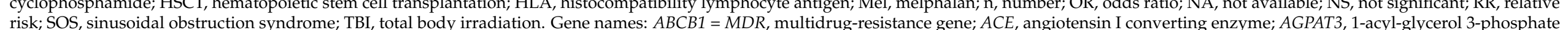

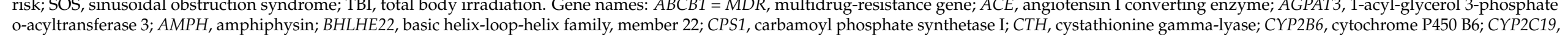

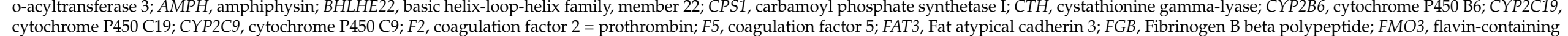

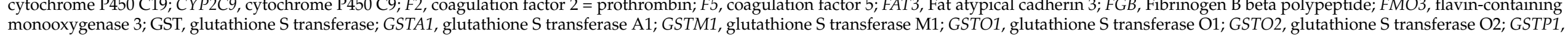

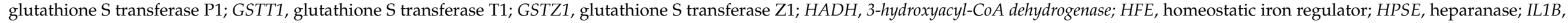

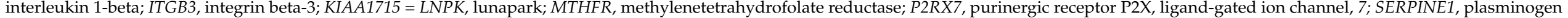
activator inhibitor-1; TPMT, thiopurine S-methyltransferase; UGT2B10, uridine diphosphate glycosyltransferase family 2 member B10; VDR, vitamin D receptor; ZNF608, Zinc Finger Protein 608. 


\subsubsection{Methylenetetrahydrofolate Reductase}

Variants in the gene coding for the methylenetetrahydrofolate reductase (MTHFR) were tested in six studies. All included studies were performed with predominantly adult patients. Goekkurt et al. [52] included 84 patients undergoing busulfan-cyclophosphamidebased allogeneic HSCT for various malignant and non-malignant diseases and identified the rs1801131(CC vs. AC/AA) or 1298A > C variant (OR 9.4, CI 1.1-81.9), but failed to show an association with the rs1801133(CC vs. CT/TT) or $677 \mathrm{C}>\mathrm{T}$ variant. Efrati et al. [33] performed an analysis on 62 patients undergoing allogeneic HSCT with a busulfan-cyclophosphamide-based conditioning regimen for acute myeloid leukemia. The authors found the rs1801131 (CC vs. AC/AA) variant (no OR published, $p=0.0002$ ) and the rs1801133(CC vs.CT/TT) variant (no OR, $p=0.0096$ ) associated with SOS. The largest study by Byun et al. [55] included 177 patients undergoing allogeneic HSCT with various conditioning regimens for different diagnoses but was limited by a low proportion of patients with SOS $(n=10 / 177,5.6 \%$ ). The authors tested the rs 1801133 (CC vs.CT /TT) variant (no OR, $p=0.089$ ) and the rs1801133(TT vs.CT/CC) variant (no OR, $p=0.234$ ) but did not find an association with SOS. Further studies in 72 to 107 patients did not identify associations of these variants with SOS $[38,40,44]$. Methotrexate was used as graft-versus-host disease prophylaxis in $>90 \%$ of patients of the studies that assessed MTHFR except in the study by Pihusch et al. [44], which also showed a very low SOS incidence $(n=3 / 89,3.4 \%)$.

\subsubsection{Other Liver Enzymes}

Kallianpur et al. [35] found an association of the hemostatic iron regulator variant rs1800562(A > G) (HFE; RR 3.7; CI 1.2-12.1) and carbamoyl phosphate synthetase I variant rs7422339(CC vs. AC/AA) (CPS1, no RR; $p=0.04$ ) with SOS in 166 adult patients undergoing autologous or allogeneic HSCT for various malignancies. Sucak et al. [50] did not identify the HFE variant rs $1799945 \mathrm{C}>\mathrm{G}$ in 102 adult patients with various underlying diseases. CPS1 was not included in further studies. The HPSE variants rs4364254 (TT vs. $\mathrm{TC} / \mathrm{CC}, p=0.004$ ) and rs4693608 (AA vs. AG/GG, $p=0.038$ ) were associated with SOS in the study by Seifert et al. [45]. Curtis et al. [46] found the cystathionine gamma-lyase (CTH) gene variant rs1021737(TT vs.GT/GG) to be associated with SOS in 76 pediatric patients undergoing busulfan-based HSCT for various malignant and non-malignant diseases (OR 10.6, CI 2.2-51.5). Variants in the flavin-containing monooxygenase 3 (FMO3) [43] and vitamin D receptor $(V D R)$ [40] were not associated with SOS.

\subsubsection{Coagulation and Vascular System}

Genes encoding coagulation system proteins were included in four identified studies. Duggan et al. and Pihusch et al. [22,44] did not find an association of prothrombin (F2) and factor V (F5) variants in mostly adult patients undergoing HSCT with varying conditioning regimens for different underlying diagnoses. The study by Pihusch et al. [44] had a low proportion of participants with SOS $(n=3 / 89,3.4 \%)$. The study also tested fibrinogen (FGB), integrin beta-3 (ITGB3), plasminogen activator inhibitor (SERPINE1), and the vasculature-associated enzyme angiotensin I-converting enzyme $(A C E)$ but did not find an association with SOS. Elbahlawan et al. [49] tested a variant in the cytokine interleukine-1 beta (IL1B) gene, which interacts with the endothelium and the coagulation system without association. Lee et al. [39] did not find an association with SOS of the purinergic receptor $P 2 X$ ligand-gated channel 7 gene (P2RX7) known to interact with interleukine- 1 in 152 mostly adult patients.

\subsubsection{Whole Exome Analysis}

The only exome-wide association study by Ansari et al. [47] in 87 pediatric patients undergoing busulfan-based allogeneic HSCT for various malignant and non-malignant diseases found eight gene variants associated with SOS in the discovery dataset, of which three were replicated in an independent cohort of 182 pediatric patients (UGT2B10, KIAA1715, BHLHE22). The uridine diphosphate glycosyltransferase 2 family, member 10 (UGT2B10, 
HR 4.7, CI 2.0-11.5) and lunapark (LNPK = KIAA1715, HR 2.7, CI 1.0-7.5) gene variants were retained in a multivariable model, which controlled for underlying disease, regimen type, and the previously identified risk variants in the GSTA1 promoter (slow metabolizer haplotypes, HR 3.1, CI 1.2-8.0).

\subsection{Investigated Genes for Association with Antineoplastic Agent Exposure}

We identified six different genes in four studies investigating $\mathrm{SOS}$ after antineoplastic treatments without HSCT (Table 3). Two studies focused on glutathione S-transferase genes: Aplenc et al. [32] assessed variants in GSTM1, GSTP1, and GSTT1 in 18 successfully genotyped adult patients receiving ozogamycin-gemtuzumab treatment for relapsed acute myeloid leukemia after HSCT. The study found no association with SOS. Vreuls et al. [48] tested 55 adult patients with metastatic colorectal cancer and oxaliplatin treatment for an association of GSTM1 and GSTT1 and found the GSTM1 "null genotype" to be associated with SOS (no OR published, $p=0.03$ ). Lennard et al. examined the TPMT $T^{*} 3 A{ }^{*} 3 B / * 3 C$ haplotypes in 203 patients [12], and Wray et al. examined the same haplotypes and MTHFR variants in 351 patients [13] undergoing acute lymphoblastic leukemia treatment. Both studies included patients from prospective trials. The former study found that the prevalence of the TPMT $* 3 A / * 3 B / * 3 C$ alleles was nearly double in the SOS cohort without evidence of association $(p=0.11)$. The other study found no evidence of association of variants in TPMT or MTHFR.

Table 3. Summary of 4 studies on genetic variants and their association with sinusoidal obstruction syndrome after antineoplastic agents without hematopoietic stem cell transplantation. Publications are listed in chronological order of publication.

\begin{tabular}{|c|c|c|c|c|c|c|c|c|c|}
\hline $\begin{array}{c}\text { Lead } \\
\text { Author, } \\
\text { Journal Year }\end{array}$ & $\begin{array}{l}\text { Study } \\
\text { Design }\end{array}$ & Location & $\begin{array}{c}\text { Population } \\
\text { (Diagnoses, Age) }\end{array}$ & $\begin{array}{l}\text { Exposure, } \\
\text { Location }\end{array}$ & $\begin{array}{c}n \\
\text { (SOS/Total) }\end{array}$ & $\begin{array}{c}\text { Genes/ } \\
\text { Re- } \\
\text { gion }\end{array}$ & $\begin{array}{c}\text { Variants } \\
\text { Investigated }\end{array}$ & OR (CI) & $p$-Value \\
\hline \multirow{5}{*}{$\begin{array}{c}\text { Aplenc } \\
\text { R, et al. Acta } \\
\text { Haematolog- } \\
\text { ica. 2003 } \\
\text { [32] }\end{array}$} & \multirow{5}{*}{$\begin{array}{l}\text { Candidate- } \\
\text { gene; } \\
\text { case-control }\end{array}$} & \multirow{5}{*}{$\begin{array}{l}\text { University } \\
\text { of } \\
\text { Washington } \\
\text { Medical } \\
\text { Center, } \\
\text { Seattle, USA }\end{array}$} & \multirow{5}{*}{$\begin{array}{l}\text { Relapsed AML; } \\
\text { mean age } \\
45.4 \text { years }\end{array}$} & \multirow{5}{*}{$\begin{array}{l}\text { Gemtuzumab for } \\
\text { relapsed disease } \\
\text { after HSCT (SOS } \\
\text { not primarily } \\
\text { associated } \\
\text { with HSCT) }\end{array}$} & \multirow{5}{*}{$\begin{array}{l}\text { 11/21 (52\%) } \\
\text { Genotyped: } \\
\text { 9/18 (50\%) }\end{array}$} & GSTM1 & $\begin{array}{c}\text { "null } \\
\text { genotype" } \ddagger\end{array}$ & - & NS \\
\hline & & & & & & GSTT1 & 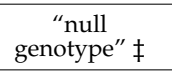 & - & NS \\
\hline & & & & & & \multirow[b]{2}{*}{ GSTP1 } & *B haplotype & OR 4 (NA) & NS \\
\hline & & & & & & & $\begin{array}{c}{ }^{*} \mathrm{C} \\
\text { haplotype }\end{array}$ & - & NS \\
\hline & & & & & & NQ01 & *2 haplotype & - & NS \\
\hline $\begin{array}{c}\text { Lennard } \\
\text { L, et al. Clin. } \\
\text { Pharmacol. } \\
\text { Ther. 2006 } \\
\text { [12] }\end{array}$ & $\begin{array}{l}\text { Candidate- } \\
\text { gene; } \\
\text { case-control } \\
\text { based on } \\
\text { prospective } \\
\text { trial }\end{array}$ & $\begin{array}{l}\text { Multicentric, } \\
\text { USA }\end{array}$ & $\begin{array}{c}\text { Acute } \\
\text { lymphoblastic } \\
\text { leukemia; median } \\
\text { age } 4 \text { years (range } \\
1-16)\end{array}$ & $\begin{array}{c}\text { Treatment } \\
\text { according to } \\
\text { protocols } \\
\text { CCG-ALL97 } \\
(n=33 / 393 \text { with } \\
\text { SOS, } 8 \%) \text { and } \\
\text { CCG-ALL99 } \\
(n=49 / 355 \text { with } \\
\text { SOS, } 14 \%)\end{array}$ & $\begin{array}{l}50 / 203 \\
(24.6 \%)\end{array}$ & TPMT & $\begin{array}{c}\text { *3A/*3C } \\
\text { haplotypes }\end{array}$ & - & $p=0.11$ \\
\hline \multirow{2}{*}{$\begin{array}{c}\text { Vreuls } \\
\text { CPH, et al. } \\
\text { Brit J Cancer. } \\
2013 \text { [48] }\end{array}$} & \multirow{2}{*}{$\begin{array}{l}\text { Candidate- } \\
\text { gene; } \\
\text { cohort }\end{array}$} & \multirow{2}{*}{$\begin{array}{l}\text { Maastricht } \\
\text { University } \\
\text { Medical } \\
\text { Centre, NL }\end{array}$} & \multirow{2}{*}{$\begin{array}{c}\text { Patients with } \\
\text { metastatic } \\
\text { colorectal cancer; } \\
\text { mean age } 62 \text { years } \\
\text { (range } 40-81 \text { ) }\end{array}$} & \multirow{2}{*}{$\begin{array}{l}\text { Initial partial } \\
\text { hepatic resection } \\
\text { and treatment } \\
\text { with oxaliplatin }\end{array}$} & \multirow{2}{*}{$32 / 55(58 \%)$} & GSTM1 & $\begin{array}{c}\text { "null } \\
\text { genotype" } \ddagger\end{array}$ & - & $p=0.026 t$ \\
\hline & & & & & & GSTT1 & $\begin{array}{c}\text { "null } \\
\text { genotype" } \ddagger\end{array}$ & - & NS \\
\hline \multirow{5}{*}{$\begin{array}{l}\text { Wray L, et al. } \\
\text { Pediatr } \\
\text { Blood } \\
\text { Cancer. } \\
2014 \text { [13] }\end{array}$} & \multirow{5}{*}{$\begin{array}{l}\text { Candidate- } \\
\text { gene; } \\
\text { prospective } \\
\text { trial }\end{array}$} & \multirow{5}{*}{$\begin{array}{l}\text { Children's } \\
\text { Hospital of } \\
\text { Philadelphia, } \\
\text { USA }\end{array}$} & \multirow{5}{*}{$\begin{array}{c}\text { Acute } \\
\text { lymphoblastic } \\
\text { leukemia; } \\
\text { pediatric patients } \\
\text { (range 1-10 years) }\end{array}$} & \multirow{5}{*}{$\begin{array}{l}\text { Treatment } \\
\text { according to } \\
\text { protocol } \\
\text { CCG-1952 }\end{array}$} & \multirow{5}{*}{$\begin{array}{l}79 / 351 \\
(22.5 \%)\end{array}$} & \multirow{3}{*}{$T P M T$} & $\begin{array}{c}\text { *3A } \\
\text { haplotype }\end{array}$ & $\begin{array}{l}\text { OR 0.7 } \\
(0.3-1.6)+\end{array}$ & NS + \\
\hline & & & & & & & $\begin{array}{c}* 3 \mathrm{~B} \\
\text { haplotype }\end{array}$ & $\begin{array}{c}\text { OR 1.0 } \\
(0.4-2.6) \dagger\end{array}$ & $\mathrm{NS}+$ \\
\hline & & & & & & & $\begin{array}{c}* 3 C \\
\text { haplotype }\end{array}$ & $\begin{array}{c}\text { OR 0.7 } \\
(0.2-1.8)+\end{array}$ & NS † \\
\hline & & & & & & \multirow{2}{*}{ MTHFR } & $\begin{array}{l}\text { rs1801133(CC } \\
\text { vs.CT/TT) }\end{array}$ & $\begin{array}{l}\text { OR } 0.9 \\
(0.3-2.4) \dagger\end{array}$ & $\mathrm{NS}+$ \\
\hline & & & & & & & $\begin{array}{l}\text { rs1801131(CC } \\
\text { vs.AC/AA) }\end{array}$ & $\begin{array}{c}\text { OR } \\
1.4(0.5-3.8)+\end{array}$ & NS + \\
\hline
\end{tabular}

Legend: bold font, significant association; $\dagger$, after adjustment for clinical covariables (multivariable regression analysis); $\ddagger$, "null genotype" is used for genotypes with absence of enzyme activity; ALL, acute lymphoblastic leukemia; allo, allogeneic; AML, acute myeloid leukemia; allo, allogeneic; BM, bone marrow; Bu, Busulfan; CI, 95\%-confidence interval; HSCT, hematopoietic stem cell transplantation; HLA, histocompatibility lymphocyte antigen; NA, not available; NS, not significant; OR, odds ratio; SOS, sinusoidal obstruction syndrome; TBI, total body irradiation. Gene names: GSTA1, glutathione S transferase A1; GSTM1, glutathione S transferase M1; GSTP1, glutathione S transferase P1; GSTT1, glutathione S transferase T1; MTHFR, methylenetetrahydrofolate reductase; NQ01, NAD(P)H Quinone Dehydrogenase 1; TPMT, thiopurine S-methyltransferase. 


\section{Discussion}

This is the first systemtic review that collected the evidence for any postulated germline genetic predictors for sinusoidal obstruction syndrome. We identified 27 studies, 23 on SOS after exposure to HSCT, and 4 after antineoplastic agents without HSCT. Three groups of genes were included in candidate-gene association studies on SOS: genes encoding (i) drug-metabolizing enzymes, mainly glutathione S-transferases (GST), cytochrome P450 family enzymes (CYP), and MTHFR; (ii) other enzymes mainly active in the liver; and (iii) coagulation factors and other proteins closely interacting with the coagulation or vascular system. Variants in nine different genes showed an association with SOS in the included candidate-gene association studies (CPS1, CTH, CYP2B6, GSTA1, GSTM1, GSTP1, HFE, HPSE, MTHFR). Of those, only two were associated in more than one study: GSTA1 in three studies [46,47,51] and MTHFR in two studies [33,52]. Additionally, the study using WES data [47] identified variants in eight genes, of which two were retained after a stepwise selection using a multivariate Cox regression model after replication in an independent cohort (UGT2B10 and KIAA1715 = LNPK).

GSTA1 variants were frequently included in genotype-phenotype association analyses due to their importance in drug metabolism, particularly in electrophilic chemotherapies such as busulfan. An association of these chemotherapies with SOS has been known for more than three decades [56]. The metabolization of busulfan is performed in the liver through conjugation with glutathione both spontaneously and by catalysis particularly of the alpha1 isoform (GSTA1), followed by mu1 (GSTM1) and pi1 (GSTP1) [57]. Three of the nine studies identified an association of GSTA1 variants with SOS, while six did not. The first reason for this discrepancy might be the differences in the genetic variants that were compared: different genetic variants in promoter regions have been shown to modify the expression of the metabolic enzyme to varying degrees [58]. Slow metabolizer haplotypes were only tested by Ansari et al. [47,51]. The slow metabolizer haplotypes were associated with SOS in multivariable association analyses taking into account underlying disease and type of conditioning regimen. Curtis et al. [46] found an association of the ${ }^{*} B^{*} B$ haplotype with SOS. Other studies compared the ${ }^{*} B$ haplotypes but did not find an association. Second, a limited number of participants included in many studies might have impacted the ability to identify associations and the precision of effect sizes with large confidence intervals. The studies reporting no association with pediatric participants included only 29 to 77 participants. Third, GSTA1 haplotypes have been consistently associated with busulfan pharmacokinetics [51,59]. However, GSTA1 was reported to be more important for busulfan clearance in young versus older children due to the maturation of other pathways for busulfan clearance, with older age rendering GSTA1 less important [60,61]. Infants also had a more variable clearance than older patients [62]. A limitation of all these studies is that busulfan clearance was not included in the models testing GSTA1 variants with SOS. Therefore, it remains unclear if GSTA1 has an association with SOS beyond its effect on busulfan clearance.

Variants in two other genes from the glutathione S-transferase family were associated with SOS in one study each, while other studies failed to report an association. GSTM1 "null genotype" was associated with SOS in one [42] study, while seven did not reveal an association $[36,37,40,41,51,52,54]$. GSTP1 $\mathrm{rs} 1695(\mathrm{~A}>\mathrm{G})$ was found associated with SOS in one study [37], while six studies showed no association [36,40,51-54]. Possibly, the heterogeneity between these studies in terms of age at HSCT, HSCT conditioning regimen, and underlying disease might have contributed to the varying results. A recent systematic review with a meta-analysis of glutathione $S$-transferase genes was performed by Kim et al. [23]. The authors included nine studies on $G S T A{ }^{*} A * A$ versus * $B$ haplotypes, seven studies on GSTM1 "null genotype", and five studies on GSTP1. They showed an association of the GSTA1*B haplotype with the area under the curve of intravenous busulfan but failed to show an association of glutathione S-transferases with SOS. However, some of the studies we identified were not included in the analysis by Kim et al. [23]. The studies by Ansari et al., 2013, 2017 and 2020 [30,47,51] were not assessed, while some 
studies that they included did not meet our inclusion criteria as they reported low patient numbers ( $<20$ participants) or no patients with the outcome of interest. While GSTM1 "null genotype" is often cited as a risk factor for SOS [7,63], our review showed that this association is inconsistently reported and needs further evaluation.

CYP2B6 variants were inconsistently associated with SOS. CYP2B6 is involved in the metabolization of the antineoplastic drug cyclophosphamide, which is often used during the conditioning regimen [64]. CYP2B6 has a variable expression between individuals due to genetic and treatment-related factors (e.g., induction by cyclophosphamide or inhibition by thiotepa). The cytochrome $\mathrm{P} 450$ gene $C Y P 2 B 6^{*} 6$ haplotype was associated with SOS in the study by Rocha et al. [40]. This was also the largest study including CYP2B6 with 107 mainly adult patients. Two other studies with 66 pediatric and 55 adult patients did not show an association of this haplotype with SOS [36,43]. These two studies included patients treated with busulfan and cyclophosphamide, while the study by Rocha et al. [40] included conditioning regimens mostly containing cyclophosphamide with other agents. The role of CYP2B6 variants in SOS remains unclear.

MTHFR variants were associated with SOS in two studies [33,52], but not in four $[38,40,44,55]$. MTHFR is coding for a key enzyme involved in the homocysteine and folate metabolism [65]. Elevated levels of homocysteine were shown to be associated with vascular injury and thrombosis [66], which provided a rationale for including MTHFR genetic variants in studies on SOS. Methotrexate is a folic acid antagonist and used as graft-versus-host disease prophylaxis in many HSCT conditioning regimens [67]. The importance of MTHFR in the folate metabolism was another reason that this gene was investigated. We found two possible explanations for the differences in associations: First, the incidence of SOS varied widely between the studies. SOS was seen in 15\% [33] to $42.9 \%$ [52] of participants in studies with, and 3.4\% [44] to $14 \%$ [40] in those without association, illustrating heterogeneity in the baseline risk for SOS in the different populations. Second, we found that studies showing an association included patients undergoing busulfan-based regimens, while studies showing no association included various conditioning regimens. In conclusion, MTHFR variants might play a role in high-risk patients and after busulfan-based conditioning.

Several other liver enzymes were analyzed in included studies. The HFE gene rs1800562(A > G) variant was associated with hemochromatosis previously, which leads to excessive iron accumulation in the liver and hepatocyte injury. That variant was associated with SOS in the study by Kallianpur et al. [35]). Sucak et al. [50] tested another hemochromatosis-associated variant $\operatorname{rs} 1799945(\mathrm{C}>\mathrm{G})$ without association with SOS. Variants in three other genes were associated with SOS in one study without testing in further studies: CTH was associated with SOS in the study by Curtis et al. [46] but a wide confidence interval indicated low precision of the estimate. $C T H$ is involved in glutathione synthesis. Glutathione is depleted by busulfan and cyclophosphamide. CPS1 codes for the enzyme necessary for the first step of the urea cycle and metabolization of excess nitrogen. It was hypothesized that the s7422339(CC vs. AC/AA) variant in CPS1 might lead to reduced antioxidant efficiency. Kallianpur et al. found an association of that variant with SOS, which was not investigated in further studies [35]. The protein coded by HPSE cleaves heparan sulfate proteoglycans, which are part of the extracellular matrix and are involved in inflammation, angiogenesis, and tissue repair. Two HPSE variants were associated with SOS in the study by Seifert et al. [45]. The importance of these genetic variants remains unclear without further replication. Genes coding for proteins relevant to the coagulation or vascular system were investigated in four studies, none of them found an association $[22,39,44,49]$.

The study by Ansari et al. 2020 [47] showed in their exome-wide analysis an association of UGT2B10 with SOS in the discovery dataset and replication in independent patients. The association remained when assessing the gene variant in different subgroups of one and multiple alkylating agents. UGT2B10 is involved in detoxifying various compounds through glucuronidation [68] and is exclusively expressed in liver tissue [69]. The other 
gene identified and replicated in the independent cohort was KIAA1715 $=$ LNPK. This gene encodes the endoplasmic reticulum (ER) junction formation factor involved in the structural organization of the endoplasmic reticulum and associated with neurodegenerative disease. LNPK was only associated with SOS in patients receiving two or more alkylating agents.

In the studies including antineoplastic agents without HSCT as exposure, two large studies by Lennard et al. [12] and Wray et al. [13] did not find an association of TPMT variants with SOS after acute lymphoblastic leukemia (ALL) treatment including patients exposed to thioguanine. The latter study did also test MTHFR variants without evidence of association. The study by Aplenc et al. [32] on 18 genotyped patients with ozogamicingemtuzumab treatment did find an OR of 4 of patients with the GSTP1*B haplotype but did not give further details on the strength of the association due to the low patient numbers. The study by Vreuls et al. [48] on 55 adult patients with metastatic colorectal cancer treated with oxaliplatin found an association with the GSTM1 "null genotype". Only histopathological criteria were used to identify SOS, which makes this study difficult to compare to others in our review.

The quality of studies included in this review was overall low, which is a limitation of the presented data in this review. There were very few studies that stratified or adjusted the analysis for ethnicity $(\mathrm{n}=2,7 \%)$, performed a power calculation for the sample size $(\mathrm{n}=2,7 \%)$, corrected results for multiple testing $(\mathrm{n}=1,4 \%)$, or replicated results in a separate sample $(n=1,4 \%)$. We then found large heterogeneity between studies. Patients varied in terms of underlying diagnoses, types of conditioning regimens or antineoplastic agents without HSCT, and outcome definitions. The age range, definition, and prevalence of outcomes varied between studies. Finally, prophylactic treatments were used in some studies in a standardized way, while most included patients with several prophylactic treatments. The heterogeneity of patients within studies was also large. Many studies included pediatric and adult patients, different underlying diagnoses, different conditioning regimens, and different prophylactic treatments in the same study sample. We estimated that the heterogeneity within and between studies is too large to perform a meta-analysis. Additionally, many gene variants were only assessed in one or only a few studies.

The strengths of our review are the broad scope and the number of studies that we identified and summarized. We applied a stringent pre-published protocol, including a data collection form, quality and bias assessment with a pre-defined threshold for high versus low-quality studies. We did not restrict our literature search for language and screened a large number of databases. We used two assessors for the screening and quality evaluation process and a third assessor for arbitration. Data collection was checked by a second author.

\section{Conclusions}

The strongest evidence for an association of genotypes with SOS was found for GSTA1 variants (slow metabolizer haplotypes). Still, it is unclear whether GSTA1 affects SOS beyond its influence on busulfan clearance. Some evidence was found for MTHFR variants in high-risk patients after busulfan-based conditioning regimens. Most included studies used a candidate-gene approach. Only one study used an exome-wide approach, which was also the only study with replication of results in an independent patient cohort. A wide number of genes was either inconsistently associated with SOS or only studied in one cohort.

Future studies should include sufficiently large samples of patients with ideally a single underlying disease using one treatment protocol. Power analyses are essential to design appropriate studies. An ideal setup are clinical trials with ancillary genetic studies using a clearly defined patient population. It is also important to adequately assess and adjust for relevant clinical covariates and ethnicity. To be able to compare future studies, standardized outcome measures should be employed. Finally, future studies 
should include replication populations that are similar to the discovery dataset to assess the external validity of identified associations.

Supplementary Materials: The following are available online at https:/ /www.mdpi.com/article/10 $.3390 / \mathrm{jpm} 11050347 / \mathrm{s} 1$. Table S1. PRISMA checklist for the systematic review of genetic predictors for sinusoidal obstruction syndrome. Table S2. Detailed search strategy to identify manuscripts of genetic risk analysis with sinusoidal obstruction syndrome (PubMed). Table S3. Quality assessment tool: Detailed description for scoring (0-12 possible points; based on STREGA checklist1 and adapted from Zazuli et al. and Leusink et al.). Table S4. Quality assessment of 27 included studies on genetic predictors for sinusoidal obstruction syndrome after HSCT or chemotherapy; after agreement was reached between authors.

Author Contributions: Conceptualization, methodology, title, abstract, and full-text screening (blinded with another assessor), data collection, data curation, formal analysis, quality and risk of bias assessment (blinded with another assessor), investigation, visualization, project administration, writing - all stages, N.W.; Methodology, data validation, formal analysis, quality and risk of bias assessment (blinded with another assessor), writing — review and editing, S.J.M.; Methodology, title, abstract, and full-text screening (blinded with another assessor), data validation, formal analysis, writing - review and editing, S.S.; Methodology, arbitration of title, abstract, and full-text screening and quality and risk of bias assessment, formal analysis, writing — review and editing, T.N.; Writingreview and editing, M.K.; Conceptualization, methodology, supervision, writing-review and editing, C.E.K., M.A.; All authors have read and agreed to the published version of the manuscript.

Funding: This research was funded by CANSEARCH Research Foundation, Geneva, Switzerland. T.N., S.J.M., S.S., and N.W. were supported by the CANSEARCH Research Foundation.

Institutional Review Board Statement: Not applicable.

Informed Consent Statement: Not applicable.

Data Availability Statement: Data sharing is not applicable to this article.

Acknowledgments: The authors thank Doris Kopp and Beatrice Minder (Institute of Social and Preventive Medicine (ISPM), University of Bern, Switzerland) for helping us to develop, refine, and execute the literature search strategy. The authors thank Chakradhara Rao S. Uppugunduri for his critical review and input on the manuscript.

Conflicts of Interest: The authors declare no conflict of interest. The funders had no role in the design of the study; in the collection, analyses, or interpretation of data; in the writing of the manuscript, or in the decision to publish the results.

\section{References}

1. Mahadeo, K.M.; Bajwa, R.; Abdel-Azim, H.; Lehmann, L.E.; Duncan, C.; Zantek, N.; Vittorio, J.; Angelo, J.; McArthur, J.; Schadler, K.; et al. Diagnosis, Grading, and Treatment Recommendations for Children, Adolescents, and Young Adults with Sinusoidal Obstructive Syndrome: An International Expert Position Statement. Lancet Haematol. 2020, 7, e61-e72. [CrossRef]

2. Fan, C.Q.; Crawford, J.M. Sinusoidal Obstruction Syndrome (Hepatic Veno-Occlusive Disease). J. Clin. Exp. Hepatol. 2014, 4, 332-346. [CrossRef] [PubMed]

3. McDonald, G.B.; Sharma, P.; Matthews, D.E.; Shulman, H.M.; Thomas, E.D. Venocclusive Disease of the Liver after Bone Marrow Transplantation: Diagnosis, Incidence, and Predisposing Factors. Hepatology 1984, 4, 116-122. [CrossRef] [PubMed]

4. Jones, R.J.; Lee, K.S.; Beschorner, W.E.; Vogel, V.G.; Grochow, L.B.; Braine, H.G.; Vogelsang, G.B.; Sensenbrenner, L.L.; Santos, G.W.; Saral, R. Venoocclusive Disease of the Liver Following Bone Marrow Transplantation. Transplantation 1987, 44, 778-783. [CrossRef]

5. Corbacioglu, S.; Carreras, E.; Ansari, M.; Balduzzi, A.; Cesaro, S.; Dalle, J.-H.; Dignan, F.; Gibson, B.; Guengoer, T.; Gruhn, B.; et al. Diagnosis and Severity Criteria for Sinusoidal Obstruction Syndrome/Veno-Occlusive Disease in Pediatric Patients: A New Classification from the European Society for Blood and Marrow Transplantation. Bone Marrow Transplant. 2018, 53, 138-145. [CrossRef]

6. Coppell, J.A.; Richardson, P.G.; Soiffer, R.; Martin, P.L.; Kernan, N.A.; Chen, A.; Guinan, E.; Vogelsang, G.; Krishnan, A.; Giralt, S.; et al. Hepatic Veno-Occlusive Disease Following Stem Cell Transplantation: Incidence, Clinical Course, and Outcome. Biol. Blood Marrow Transplant. 2010, 16, 157-168. [CrossRef] [PubMed]

7. Dalle, J.-H.; Giralt, S.A. Hepatic Veno-Occlusive Disease after Hematopoietic Stem Cell Transplantation: Risk Factors and Stratification, Prophylaxis, and Treatment. Biol. Blood Marrow Transplant. 2016, 22, 400-409. [CrossRef] 
8. McDonald, G.B.; Hinds, M.S.; Fisher, L.D.; Schoch, H.G.; Wolford, J.L.; Banaji, M.; Hardin, B.J.; Shulman, H.M.; Clift, R.A. Veno-Occlusive Disease of the Liver and Multiorgan Failure after Bone Marrow Transplantation: A Cohort Study of 355 Patients. Ann. Intern. Med. 1993, 118, 255-267. [CrossRef]

9. Corbacioglu, S.; Cesaro, S.; Faraci, M.; Valteau-Couanet, D.; Gruhn, B.; Rovelli, A.; Boelens, J.J.; Hewitt, A.; Schrum, J.; Schulz, A.S.; et al. Defibrotide for Prophylaxis of Hepatic Veno-Occlusive Disease in Paediatric Haemopoietic Stem-Cell Transplantation: An Open-Label, Phase 3, Randomised Controlled Trial. Lancet 2012, 379, 1301-1309. [CrossRef]

10. Choi, J.-H.; Won, Y.-W.; Kim, H.S.; Oh, Y.-H.; Lim, S.; Kim, H.-J. Oxaliplatin-Induced Sinusoidal Obstruction Syndrome Mimicking Metastatic Colon Cancer in the Liver. Oncol. Lett. 2016, 11, 2861-2864. [CrossRef]

11. Koo, S.X.; Chan, S.H.; Ngeow, J. Genetic Predisposition Resulting in Sinusoidal Obstruction Syndrome in a Patient with Resected Sigmoid Cancer on Adjuvant Oxaliplatin. BMJ Case Rep. 2016, 2016. [CrossRef]

12. Lennard, L.; Richards, S.; Cartwright, C.S.; Mitchell, C.; Lilleyman, J.S.; Vora, A. UK MRC/NCRI Childhood Leukaemia Working Party The Thiopurine Methyltransferase Genetic Polymorphism Is Associated with Thioguanine-Related Veno-Occlusive Disease of the Liver in Children with Acute Lymphoblastic Leukemia. Clin. Pharm. 2006, 80, 375-383. [CrossRef]

13. Wray, L.; Vujkovic, M.; McWilliams, T.; Cannon, S.; Devidas, M.; Stork, L.; Aplenc, R. TPMT and MTHFR Genotype Is Not Associated with Altered Risk of Thioguanine-Related Sinusoidal Obstruction Syndrome in Pediatric Acute Lymphoblastic Leukemia: A Report from the Children's Oncology Group. Pediatr. Blood Cancer 2014, 61, 2086-2088. [CrossRef] [PubMed]

14. Valla, D.-C.; Cazals-Hatem, D. Sinusoidal Obstruction Syndrome. Clin. Res. Hepatol. Gastroenterol. 2016, 40, 378-385. [CrossRef]

15. Kizilocak, H.; Dikme, G.; Özdemir, N.; Kuruğoğlu, S.; Adaletli, İ.; Erkan, T.; Celkan, T. Sinusoidal Obstruction Syndrome During Chemotherapy of Pediatric Cancers and Its Successful Management With Defibrotide. J. Pediatr. Hematol. Oncol. 2017, 39, e373-e376. [CrossRef] [PubMed]

16. Mohty, M.; Malard, F.; Abecassis, M.; Aerts, E.; Alaskar, A.S.; Aljurf, M.; Arat, M.; Bader, P.; Baron, F.; Bazarbachi, A.; et al. Sinusoidal Obstruction Syndrome/Veno-Occlusive Disease: Current Situation and Perspectives-A Position Statement from the European Society for Blood and Marrow Transplantation (EBMT). Bone Marrow Transplant. 2015, 50, 781-789. [CrossRef]

17. Qiao, J.; Fu, J.; Fang, T.; Huang, Y.; Mi, H.; Yang, N.; Chen, C.; Xu, K.; Zeng, L. Evaluation of the Effects of Preconditioning Regimens on Hepatic Veno-Occlusive Disease in Mice after Hematopoietic Stem Cell Transplantation. Exp. Mol. Pathol. 2015, 98, 73-78. [CrossRef]

18. Park, Y.D.; Yasui, M.; Yoshimoto, T.; Chayama, K.; Shimono, T.; Okamura, T.; Inoue, M.; Yumura-Yagi, K.; Kawa-Ha, K. Changes in Hemostatic Parameters in Hepatic Veno-Occlusive Disease Following Bone Marrow Transplantation. Bone Marrow Transplant. 1997, 19, 915-920. [CrossRef]

19. Carreras, E. Veno-Occlusive Disease of the Liver after Hemopoietic Cell Transplantation. Eur. J. Haematol. 2000, 64, 281-291. [CrossRef]

20. Richardson, P.; Aggarwal, S.; Topaloglu, O.; Villa, K.F.; Corbacioglu, S. Systematic Review of Defibrotide Studies in the Treatment of Veno-Occlusive Disease/Sinusoidal Obstruction Syndrome (VOD/SOS). Bone Marrow Transplant. 2019, 54, 19511962. [CrossRef] [PubMed]

21. Piccin, A.; Sartori, M.T.; Bisogno, G.; Van Schilfgaarde, M.; Saggiorato, G.; Pierro, A.M.D.; Corvetta, D.; Marcheselli, L.; Andrea, M.; Gastl, G.; et al. New Insights into Sinusoidal Obstruction Syndrome. Intern. Med. J. 2017, 47, 1173-1183. [CrossRef] [PubMed]

22. Duggan, C.; Schmidt, M.; Lawler, M.; White, B.; Cusack, S.; McCann, S.; Smith, O. The Prothrombin Gene Variant G20210A but Not Factor V Leiden May Be Associated with Veno-Occlusive Disease Following BMT. Bone Marrow Transplant. 1999, 24, 693-694. [CrossRef]

23. Kim, M.G.; Kwak, A.; Choi, B.; Ji, E.; Oh, J.M.; Kim, K. Effect of Glutathione S-Transferase Genetic Polymorphisms on Busulfan Pharmacokinetics and Veno-Occlusive Disease in Hematopoietic Stem Cell Transplantation: A Meta-Analysis. Basic Clin. Pharmacol. Toxicol. 2019, 124, 691-703. [CrossRef]

24. Moher, D.; Shamseer, L.; Clarke, M.; Ghersi, D.; Liberati, A.; Petticrew, M.; Shekelle, P.; Stewart, L.A. Preferred Reporting Items for Systematic Review and Meta-Analysis Protocols (PRISMA-P) 2015 Statement. Syst. Rev. 2015, 4, 1. [CrossRef] [PubMed]

25. Ouzzani, M.; Hammady, H.; Fedorowicz, Z.; Elmagarmid, A. Rayyan-A Web and Mobile App for Systematic Reviews. Syst. Rev. 2016, 5, 210. [CrossRef] [PubMed]

26. Zazuli, Z.; Vijverberg, S.; Slob, E.; Liu, G.; Carleton, B.; Veltman, J.; Baas, P.; Masereeuw, R.; Maitland-van der Zee, A.-H. Genetic Variations and Cisplatin Nephrotoxicity: A Systematic Review. Front. Pharm. 2018, 9, 1111. [CrossRef] [PubMed]

27. Little, J.; Higgins, J.P.T.; Ioannidis, J.P.A.; Moher, D.; Gagnon, F.; von Elm, E.; Khoury, M.J.; Cohen, B.; Davey-Smith, G.; Grimshaw, J.; et al. Strengthening the Reporting of Genetic Association Studies (STREGA): An Extension of the Strengthening the Reporting of Observational Studies in Epidemiology (STROBE) Statement. J. Clin. Epidemiol. 2009, 62, 597-608.e4. [CrossRef]

28. Vandenbroucke, J.; von Elm, E.; Altman, D.; Gøtzsche, P.; Mulrow, C.; Pocock, S.; Poole, C.; Schlesselman, J.; Egger, M. Strengthening the Reporting of Observational Studies in Epidemiology (STROBE). Epidemiology 2007, 18, 805-835. [CrossRef]

29. Ansari, M.; Lauzon-Joset, J.-F.; Vachon, M.-F.; Duval, M.; Théoret, Y.; Champagne, M.A.; Krajinovic, M. Influence of GST Gene Polymorphisms on Busulfan Pharmacokinetics in Children. Bone Marrow Transplant. 2010, 45, 261-267. [CrossRef]

30. Ansari, M.; Rezgui, M.A.; Théoret, Y.; Uppugunduri, C.R.S.; Mezziani, S.; Vachon, M.-F.; Desjean, C.; Rousseau, J.; Labuda, M.; Przybyla, C.; et al. Glutathione S-Transferase Gene Variations Influence BU Pharmacokinetics and Outcome of Hematopoietic SCT in Pediatric Patients. Bone Marrow Transplant. 2013, 48, 939-946. [CrossRef] 
31. Piao, Z.; Kim, H.J.; Choi, J.Y.; Hong, C.R.; Lee, J.W.; Kang, H.J.; Park, K.D.; Shin, H.Y. Effect of FOXP3 Polymorphism on the Clinical Outcomes after Allogeneic Hematopoietic Stem Cell Transplantation in Pediatric Acute Leukemia Patients. Int. Immunopharmacol. 2016, 31, 132-139. [CrossRef] [PubMed]

32. Aplenc, R.; Vachani, A.; Han, P.; Glatfelter, W.; Sievers, E.L. Pharmacogenetics of Gemtuzumab-Associated Hepatic Sinusoidal Occlusion Syndrome after Hematopoietic Stem Cell Transplant. Acta Haematol. 2003, 110, 207-210. [CrossRef] [PubMed]

33. Efrati, E.; Zuckerman, T.; Ben-Ami, E.; Krivoy, N. MTHFR C677T/A1298C Genotype: A Possible Risk Factor for Liver Sinusoidal Obstruction Syndrome. Bone Marrow Transplant. 2014, 49, 726-727. [CrossRef] [PubMed]

34. Elmaagacli, A.H.; Koldehoff, M.; Steckel, N.K.; Trenschel, R.; Ottinger, H.; Beelen, D.W. Cytochrome P 450 2C19 Loss-of-Function Polymorphism Is Associated with an Increased Treatment-Related Mortality in Patients Undergoing Allogeneic Transplantation. Bone Marrow Transplant. 2007, 40, 659-664. [CrossRef] [PubMed]

35. Kallianpur, A.R.; Hall, L.D.; Yadav, M.; Byrne, D.W.; Speroff, T.; Dittus, R.S.; Haines, J.L.; Christman, B.W.; Summar, M.L. The Hemochromatosis C282Y Allele: A Risk Factor for Hepatic Veno-Occlusive Disease after Hematopoietic Stem Cell Transplantation. Bone Marrow Transplant. 2005, 35, 1155-1164. [CrossRef]

36. Terakura, S.; Onizuka, M.; Fukumoto, M.; Kuwatsuka, Y.; Kohno, A.; Ozawa, Y.; Miyamura, K.; Inagaki, Y.; Sawa, M.; Atsuta, Y.; et al. Analysis of Glutathione S-Transferase and Cytochrome P450 Gene Polymorphism in Recipients of Dose-Adjusted Busulfan-Cyclophosphamide Conditioning. Int. J. Hematol. 2020, 111, 84-92. [CrossRef]

37. Krivoy, N.; Zuckerman, T.; Elkin, H.; Froymovich, L.; Rowe, J.M.; Efrati, E. Pharmacokinetic and Pharmacogenetic Analysis of Oral Busulfan in Stem Cell Transplantation: Prediction of Poor Drug Metabolism to Prevent Drug Toxicity. Curr. Drug Saf. 2012, 7, 211-217. [CrossRef]

38. Kim, I.; Lee, K.-H.; Kim, J.H.; Ra, E.K.; Yoon, S.-S.; Hong, Y.-C.; Park, S.S.; Kim, C.S.; Park, S.; Kim, B.K. Polymorphisms of the Methylenetetrahydrofolate Reductase Gene and Clinical Outcomes in HLA-Matched Sibling Allogeneic Hematopoietic Stem Cell Transplantation. Ann. Hematol. 2007, 86, 41-48. [CrossRef] [PubMed]

39. Lee, K.-H.; Park, S.S.; Kim, I.; Kim, J.H.; Ra, E.K.; Yoon, S.-S.; Hong, Y.-C.; Park, S.; Kim, B.K. P2X7 Receptor Polymorphism and Clinical Outcomes in HLA-Matched Sibling Allogeneic Hematopoietic Stem Cell Transplantation. Haematologica 2007, 92, 651-657. [CrossRef]

40. Rocha, V.; Porcher, R.; Fernandes, J.F.; Filion, A.; Bittencourt, H.; Silva, W.; Vilela, G.; Zanette, D.L.; Ferry, C.; Larghero, J.; et al. Association of Drug Metabolism Gene Polymorphisms with Toxicities, Graft-versus-Host Disease and Survival after HLAIdentical Sibling Hematopoietic Stem Cell Transplantation for Patients with Leukemia. Leukemia 2009, 23, 545-556. [CrossRef]

41. Ansari, M.; Huezo-Diaz, P.; Rezgui, M.A.; Marktel, S.; Duval, M.; Bittencourt, H.; Cappelli, B.; Krajinovic, M. Influence of Glutathione S-Transferase Gene Polymorphisms on Busulfan Pharmacokinetics and Outcome of Hematopoietic Stem-Cell Transplantation in Thalassemia Pediatric Patients. Bone Marrow Transplant. 2016, 51, 377-383. [CrossRef]

42. Srivastava, A.; Poonkuzhali, B.; Shaji, R.V.; George, B.; Mathews, V.; Chandy, M.; Krishnamoorthy, R. Glutathione S-Transferase M1 Polymorphism: A Risk Factor for Hepatic Venoocclusive Disease in Bone Marrow Transplantation. Blood 2004, 104, 1574-1577. [CrossRef]

43. Uppugunduri, C.R.S.; Rezgui, M.A.; Diaz, P.H.; Tyagi, A.K.; Rousseau, J.; Daali, Y.; Duval, M.; Bittencourt, H.; Krajinovic, M.; Ansari, M. The Association of Cytochrome P450 Genetic Polymorphisms with Sulfolane Formation and the Efficacy of a Busulfan-Based Conditioning Regimen in Pediatric Patients Undergoing Hematopoietic Stem Cell Transplantation. Pharm. J. 2014, 14, 263-271. [CrossRef]

44. Pihusch, M.; Lohse, P.; Reitberger, J.; Hiller, E.; Andreesen, R.; Kolb, H.-J.; Holler, E.; Pihusch, R. Impact of Thrombophilic Gene Mutations and Graft-versus-Host Disease on Thromboembolic Complications after Allogeneic Hematopoietic Stem-Cell Transplantation. Transplantation 2004, 78, 911-918. [CrossRef]

45. Seifert, C.; Wittig, S.; Arndt, C.; Gruhn, B. Heparanase Polymorphisms: Influence on Incidence of Hepatic Sinusoidal Obstruction Syndrome in Children Undergoing Allogeneic Hematopoietic Stem Cell Transplantation. J. Cancer Res. Clin. Oncol. 2015, 141, 877-885. [CrossRef] [PubMed]

46. Huezo-Diaz Curtis, P.; Uppugunduri, C.R.S.; Muthukumaran, J.; Rezgui, M.A.; Peters, C.; Bader, P.; Duval, M.; Bittencourt, H.; Krajinovic, M.; Ansari, M. Association of CTH Variant with Sinusoidal Obstruction Syndrome in Children Receiving Intravenous Busulfan and Cyclophosphamide before Hematopoietic Stem Cell Transplantation. Pharm. J. 2016. [CrossRef] [PubMed]

47. Ansari, M.; Petrykey, K.; Rezgui, M.A.; Del Vecchio, V.; Cortyl, J.; Ralph, R.-O.; Nava, T.; Beaulieu, P.; St-Onge, P.; Jurkovic Mlakar, S.; et al. Genetic Susceptibility to Hepatic Sinusoidal Obstruction Syndrome in Pediatric Patients Undergoing Hematopoietic Stem Cell Transplantation. Biol. Blood Marrow Transplant. 2020, 26, 920-927. [CrossRef] [PubMed]

48. Vreuls, C.P.H.; Olde Damink, S.W.M.; Koek, G.H.; Winstanley, A.; Wisse, E.; Cloots, R.H.E.; van den Broek, M.a.J.; Dejong, C.H.C.; Bosman, F.T.; Driessen, A. Glutathione S-Transferase M1-Null Genotype as Risk Factor for SOS in Oxaliplatin-Treated Patients with Metastatic Colorectal Cancer. Br. J. Cancer 2013, 108, 676-680. [CrossRef]

49. Elbahlawan, L.; McArthur, J.; Quasney, M.W.; Pei, D.; Srivastava, K.; Dahmer, M.K.; Barfield, R. Association of IL-1 $\beta$-511 Polymorphism with Severe Veno-Occlusive Disease in Pediatric-Matched Allogeneic Hematopoietic Stem Cell Transplantation. J. Pediatr. Hematol. Oncol. 2012, 34, 175-179. [CrossRef] [PubMed]

50. Sucak, G.T.; Yaşar, D.G.; Yegin, Z.A.; Ergün, M.A.; Ozkurt, Z.N.; Aki, Ş.Z.; Güntekin, S. The Prognostic Role of Hemochromatosis H63D Allele in Allogeneic Hematopoietic Stem Cell Transplantation. Ann. Hematol. 2012, 91, 1281-1287. [CrossRef] 
51. Ansari, M.; Curtis, P.H.-D.; Uppugunduri, C.R.S.; Rezgui, M.A.; Nava, T.; Mlakar, V.; Lesne, L.; Théoret, Y.; Chalandon, Y.; Dupuis, L.L.; et al. GSTA1 Diplotypes Affect Busulfan Clearance and Toxicity in Children Undergoing Allogeneic Hematopoietic Stem Cell Transplantation: A Multicenter Study. Oncotarget 2017, 8, 90852-90867. [CrossRef] [PubMed]

52. Goekkurt, E.; Stoehlmacher, J.; Stueber, C.; Wolschke, C.; Eiermann, T.; Iacobelli, S.; Zander, A.R.; Ehninger, G.; Kröger, N. Pharmacogenetic Analysis of Liver Toxicity after Busulfan/Cyclophosphamide-Based Allogeneic Hematopoietic Stem Cell Transplantation. Anticancer Res. 2007, 27, 4377-4380. [PubMed]

53. Zwaveling, J.; Press, R.R.; Bredius, R.G.M.; van Derstraaten, T.R.J.H.M.; den Hartigh, J.; Bartelink, I.H.; Boelens, J.J.; Guchelaar, H.-J. Glutathione S-Transferase Polymorphisms Are Not Associated with Population Pharmacokinetic Parameters of Busulfan in Pediatric Patients. Drug Monit 2008, 30, 504-510. [CrossRef] [PubMed]

54. Johnson, L.; Orchard, P.J.; Baker, K.S.; Brundage, R.; Cao, Q.; Wang, X.; Langer, E.; Farag-El Maasah, S.; Ross, J.A.; Remmel, R.; et al. Glutathione S-Transferase A1 Genetic Variants Reduce Busulfan Clearance in Children Undergoing Hematopoietic Cell Transplantation. J. Clin. Pharm. 2008, 48, 1052-1062. [CrossRef]

55. Byun, J.M.; Kim, H.-L.; Shin, D.-Y.; Koh, Y.; Yoon, S.-S.; Seong, M.-W.; Park, S.S.; Kim, J.H.; Lee, Y.-G.; Kim, I. The Impact of Methylenetetrahydrofolate Reductase C677T Polymorphism on Patients Undergoing Allogeneic Hematopoietic Stem Cell Transplantation with Methotrexate Prophylaxis. PLoS ONE 2016, 11, e0163998. [CrossRef] [PubMed]

56. Morgan, M.; Dodds, A.; Atkinson, K.; Szer, J.; Downs, K.; Biggs, J. The Toxicity of Busulphan and Cyclophosphamide as the Preparative Regimen for Bone Marrow Transplantation. Br. J. Haematol. 1991, 77, 529-534. [CrossRef]

57. Czerwinski, M.; Gibbs, J.P.; Slattery, J.T. Busulfan Conjugation by Glutathione S-Transferases Alpha, Mu, and Pi. Drug Metab. Dispos. 1996, 24, 1015-1019.

58. Coles, B.F.; Morel, F.; Rauch, C.; Huber, W.W.; Yang, M.; Teitel, C.H.; Green, B.; Lang, N.P.; Kadlubar, F.F. Effect of Polymorphism in the Human Glutathione S-Transferase A1 Promoter on Hepatic GSTA1 and GSTA2 Expression. Pharmacogenetics 2001, 11, 663-669. [CrossRef]

59. Nava, T.; Rezgui, M.A.; Uppugunduri, C.R.S.; Curtis, P.H.-D.; Théoret, Y.; Duval, M.; Daudt, L.E.; Ansari, M.; Krajinovic, M.; Bittencourt, H. GSTA1 Genetic Variants and Conditioning Regimen: Missing Key Factors in Dosing Guidelines of Busulfan in Pediatric Hematopoietic Stem Cell Transplantation. Biol. Blood Marrow Transplant. 2017, 23, 1918-1924. [CrossRef]

60. Ten Brink, M.H.; van Bavel, T.; Swen, J.J.; Straaten, T.; Bredius, R.G.; Lankester, A.C.; Zwaveling, J.; Guchelaar, H.-J. Effect of Genetic Variants GSTA1 and CYP39A1 and Age on Busulfan Clearance in Pediatric Patients Undergoing Hematopoietic Stem Cell Transplantation. Pharmacogenomics 2013, 14, 1683-1690. [CrossRef]

61. Kodidela, S.; Kumar, S.S.; Uppugunduri, C.R.S. Developmental Pattern of Hepatic Drug-Metabolizing Enzymes in Pediatric Population and Its Role in Optimal Drug Treatment. Arch. Med. Health Sci. 2017, 5, 8.

62. McCune, J.S.; Bemer, M.J.; Barrett, J.S.; Scott Baker, K.; Gamis, A.S.; Holford, N.H.G. Busulfan in Infant to Adult Hematopoietic Cell Transplant Recipients: A Population Pharmacokinetic Model for Initial and Bayesian Dose Personalization. Clin. Cancer Res. 2014, 20, 754-763. [CrossRef]

63. Corbacioglu, S.; Jabbour, E.J.; Mohty, M. Risk Factors for Development of and Progression of Hepatic Veno-Occlusive Disease/Sinusoidal Obstruction Syndrome. Biol. Blood Marrow Transplant. 2019, 25, 1271-1280. [CrossRef]

64. Zanger, U.M.; Klein, K. Pharmacogenetics of Cytochrome P450 2B6 (CYP2B6): Advances on Polymorphisms, Mechanisms, and Clinical Relevance. Front. Genet. 2013, 4. [CrossRef]

65. Goyette, P.; Sumner, J.S.; Milos, R.; Duncan, A.M.V.; Rosenblatt, D.S.; Matthews, R.G.; Rozen, R. Human Methylenetetrahydrofolate Reductase: Isolation of CDNA, Mapping and Mutation Identification. Nat. Genet. 1994, 7, 195-200. [CrossRef] [PubMed]

66. Prasad, K. Homocysteine, a Risk Factor for Cardiovascular Disease. Int. J. Angiol. 1999, 8, 76-86. [CrossRef] [PubMed]

67. Storb, R.; Deeg, H.J.; Whitehead, J.; Appelbaum, F.; Beatty, P.; Bensinger, W.; Buckner, C.D.; Clift, R.; Doney, K.; Farewell, V. Methotrexate and Cyclosporine Compared with Cyclosporine Alone for Prophylaxis of Acute Graft versus Host Disease after Marrow Transplantation for Leukemia. N. Engl. J. Med. 1986, 314, 729-735. [CrossRef]

68. Kato, M.; Ishimaru, S.; Seki, M.; Yoshida, K.; Shiraishi, Y.; Chiba, K.; Kakiuchi, N.; Sato, Y.; Ueno, H.; Tanaka, H.; et al. Long-Term Outcome of 6-Month Maintenance Chemotherapy for Acute Lymphoblastic Leukemia in Children. Leukemia 2017, 31, 580-584. [CrossRef] [PubMed]

69. Fagerberg, L.; Hallström, B.M.; Oksvold, P.; Kampf, C.; Djureinovic, D.; Odeberg, J.; Habuka, M.; Tahmasebpoor, S.; Danielsson, A.; Edlund, K.; et al. Analysis of the Human Tissue-Specific Expression by Genome-Wide Integration of Transcriptomics and Antibody-Based Proteomics. Mol. Cell Proteom. 2014, 13, 397-406. [CrossRef] [PubMed] 